\title{
Magnetically-induced buckling of a whirling conducting rod with applications to electrodynamic space tethers
}

\author{
J. Valverde \\ Department of Civil and Environmental Engineering, \\ University of California, Berkeley, USA \\ G.H.M. van der Heijden* \\ Centre for Nonlinear Dynamics, University College London, \\ Gower Street, London WC1E 6BT, UK
}

(Dated: October 31, 2018)

\begin{abstract}
We study the effect of a magnetic field on the behaviour of a slender conducting elastic structure, motivated by stability problems of electrodynamic space tethers. Both statical (buckling) and dynamical (whirling) instability are considered and we also compute post-buckling configurations. The equations used are the geometrically exact Kirchhoff equations. Magnetic buckling of a welded rod is found to be described by a surprisingly degenerate bifurcation, which is unfolded when both transverse anisotropy of the rod and angular velocity are considered. By solving the linearised equations about the (quasi-) stationary solutions we find various secondary instabilities. Our results are relevant for current designs of electrodynamic space tethers and potentially for future applications in nano- and molecular wires.
\end{abstract}

Keywords: rod mechanics, Kirchhoff equations, magnetic buckling, degenerate pitchfork bifurcations, Hopf bifurcation, spinning electrodynamic tether

*Electronic address: g.heijden@ucl.ac.uk 


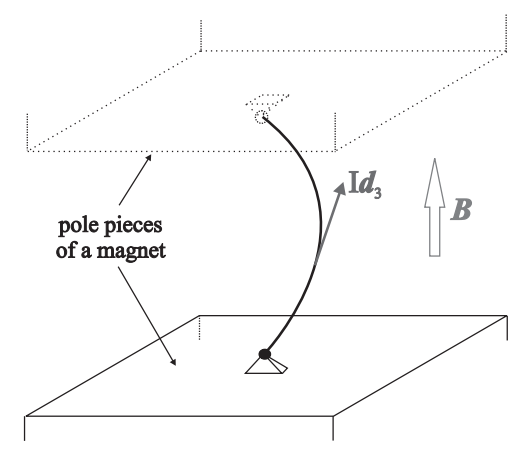

FIG. 1: Experimental setup for a conducting wire.

\section{INTRODUCTION}

A straight current-carrying wire held in tension between pole faces of a magnet is well known to buckle into a (roughly) helical configuration at a critical current (see Figure 1). A photograph of this phenomenon is shown in Section 10.4.3 of [24], where a linear stability analysis is carried out for a simple string model. (A string is here meant to be a perfectly flexible elastic wire.) The problem was studied by Wolfe [21] by means of a rigorous bifurcation analysis for a (nonlinearly elastic) string suspended between fixed supports and placed in a uniform magnetic field directed parallel to the undeformed wire. He found that an infinite number of solution branches bifurcate from the trivial straight solution, much like in the Euler elastica under compressive load. Minimisation of the potential energy indicated that the first branch of bifurcating solutions is stable while all other branches are unstable.

In a subsequent paper Wolfe [22] extends the analysis to a uniformly rotating (whirling) string and shows again the existence of bifurcating branches of whirling non-trivial solutions. This result was further extended by Healey [7] using equivariant bifurcation theory in order to deal with the symmetries of the problem, which caused the bifurcations to be degenerate.

Wolfe also considered a conducting rod in a uniform magnetic field [23]. In addition to extension a rod can undergo flexure, torsion and shear, and for the case of welded boundary conditions it was found that in certain cases bifurcation occurs, with the usual infinity of non-trivial equilibrium states. All the works cited above were content with showing the existence of bifurcating solutions and did not study their post-buckling behaviour. The Hamiltonian structure of the equations for a rod in a magnetic field was investigated in [17] where it was shown that in the case of an isotropic, inextensible and unshearable rod the 
equations are completely integrable.

The study of strings and rods in a magnetic field is of great interest to space tethers. Although space tethers in the last 20 years or so have become a well established concept in astrodynamics [2], new designs continue to be proposed that hold great potential for future space applications. A space tether is a long cable used to connect spacecraft to each other or to other orbiting bodies such as space stations, boosters, payloads, etc. in order to transfer energy and momentum thus providing space propulsion without consuming propellant. These tethers have been studied as elastic strings (e.g., [10]) and as dumbbell systems (e.g., [25]). An important class of space tethers is formed by the so-called electrodynamic tethers (ETs). These employ the earth's magnetic field and ionospheric plasma to generate a current, according to Faraday's Law, and hence thrust or drag forces without expending chemical fuel. An example is the Short Electrodynamic Tether (SET) prototype of the European Space Agency [18, 19]. This tether system, which comprises a central module from which two tethers each about a hundred metres long extend, is designed to operate at high inclination and in low orbit. Due to the shape of the earth's magnetic field this means that the desirable orientation of the tether is the horizontal one (i.e., with the axis of smallest moment of inertia normal to the orbit plane). The gravity gradient ordinarily causes a tether to drift to the stable radial position. Therefore, in order to keep the system in the horizontal position, an axial spin velocity is applied for gyroscopic stability [8, 15, 18]. This requires significant torsional and bending rigidity of the tether which therefore has to be modelled as a rod, not a string. The applied spin causes large deformations that present stability issues similar to those in an unbalanced rotor system, which have been studied in previous work [19, 20].

In this paper we apply large-deformation rod theory to study the effect of a magnetic field on the behaviour of a slender conducting elastic structure possibly subject to end forces. Both statical (buckling) and dynamical (whirling) instability are considered and we also compute post-buckling configurations. The work extends the stability analysis of the SET in [19] by including the effect of the magnetic field. This effect was considered small for the SET but that need not be true for other tether designs. For instance, very long and flexible tethers subject to boundary conditions that are not too restraining (e.g., no big end masses) might well operate in the region of the first magnetic buckling instability.

We consider welded boundary conditions. These are appropriate for tethers with suffi- 
ciently large attached end masses (see Figure 2). Unlike in string buckling the rod does not require a tensile force in the trivial state, but we allow for such an applied force as well. The pertinent dimensionless parameter that governs buckling measures the product of current and magnetic field against the bending force. We find that magnetic buckling of the welded rod is described by a remarkably degenerate pitchfork bifurcation. Wolfe considered welded boundary conditions in his statical study in [23] and encountered degeneracies (evendimensional eigenspaces) because of rotational symmetry of the problem, but we show that further complications occur, involving branches connecting the bifurcating branches.

We also study steady whirling solutions for which we introduce a rotating coordinate system. This extends Wolfe's analysis of whirling strings to whirling rods. It is found that applied spin resolves the degeneracies of the pitchfork bifurcations, provided that the rod is transversely anisotropic. We perform a stability analysis by computing eigenvalues of the linearised boudary-value problem about a (quasi-) stationary solution. For this we use a continuation (or homotopy) approach that takes advantage of the fact that exact expressions for the (imaginary) eigenvalues can be obtained in an appropriate limit (no spin, no magnetic field). The eigenvalues in this limit are then traced as system parameters are varied.

The paper is organised as follows. First, in Section 2, we give more details about the tether application and discuss the effect of the earth's magnetic field on an electrodynamic tether. Then we present our rod mechanics formulation in Section 3, in which the magnetic field enters the force balance equation through the Lorentz body force. For the study of whirling solutions the equilibrium equations are transformed to a coordinate system rotating at constant angular velocity (Section 4). The linearisation is presented in Section 5 and our continuation approach to stability analysis is discussed in Section 6. Results are presented in the form of bifurcation diagrams in Section 7, both for the statical and dynamical case. We also pick up some secondary bifurcations with associated loss of stability. Finally, in Section 8 we comment on the implications of our results for electrodynamic tethers and draw some conclusions.

\section{ELECTRODYNAMIC TETHERS AND THE EARTH'S MAGNETIC FIELD}

Electrodynamic tethers are electrical conductors that interact with the geomagnetic field

in such a way that an electromotive force (e.m.f.) is generated along the tether due to 


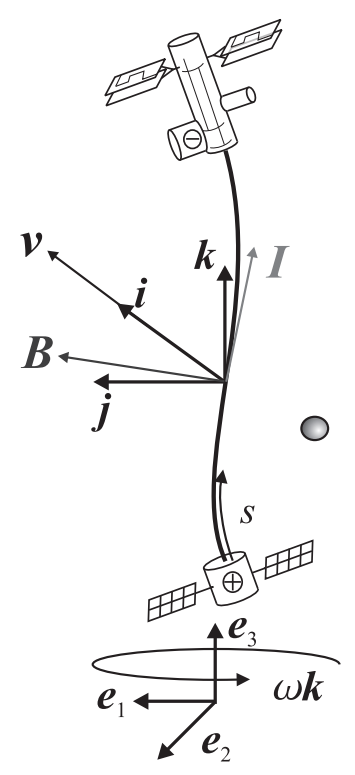

FIG. 2: An electrodynamic tether in the Earth's magnetic field.

Faraday's Law [11]. The electrical circuit is closed by means of two contactors attached to the ends of the tether which interact with the surrounding ionospheric plasma and allow a current to flow.

Figure 2 shows an electrodynamic tether connecting two satellites. Let $\{\boldsymbol{i}, \boldsymbol{j}, \boldsymbol{k}\}$ be the orbital frame, which may be assumed to be inertial. This is due to the fact that external forces on the tether system, that is, electrodynamic forces, are small enough to consider a circular Keplerian orbit which allows to decouple translational orbital dynamics from structural deformation dynamics [11]. Besides, since the angular velocity of the tether around the earth is much smaller than the typical spin velocity about its axis, rotational orbital dynamics can also be decoupled from structural dynamics of the tether subjected to spin rotation, which is the main issue of the present paper [19]. In such a case, the tether is assumed to travel with a velocity $\boldsymbol{v}$ in the direction of $\boldsymbol{i}$. The e.m.f. between the ends induced by this motion is given by

$$
E=\int_{l}\left(\boldsymbol{v} \times \boldsymbol{B}_{0}\right) \cdot d \boldsymbol{l}
$$

where $\boldsymbol{B}_{0}$ is the magnetic field and $d \boldsymbol{l}$ is a differential along the length of the tether. Because the tether is part of a closed circuit a current $\boldsymbol{I}$ will flow in the direction of increasing $E$ and the system functions as a generator. This current in turn gives rise to a Lorentz force 
$\boldsymbol{F}_{L}$ through

$$
d \boldsymbol{F}_{L}=d \boldsymbol{I} \times \boldsymbol{B}_{0}
$$

This force can be used to drag the system without expending chemical fuel [2, 11]. Alternatively, if a current is forced against the e.m.f. the system becomes a motor boosting itself to a higher orbit.

If we denote the position co-ordinates of the tether's central axis relative to $\{\boldsymbol{i}, \boldsymbol{j}, \boldsymbol{k}\}$ by $(X, Y, Z)$, then the current vector, which is directed along the tangent of the tether, can be expressed as

$$
d \boldsymbol{I}=I d \boldsymbol{l}=I\left(\frac{\partial X}{\partial s}, \frac{\partial Y}{\partial s}, \frac{\partial Z}{\partial s}\right)^{T} d s .
$$

The maximum force is generated when $\boldsymbol{I}$ and $\boldsymbol{B}_{0}$ are perpendicular. In the ET operation conditions both vectors will in general not be perpendicular because the tether is not perfectly straight and the magnetic field lines will not be perpendicular to the tether over its entire length. In order to represent this imperfection let us therefore assume that the magnetic field has an extra component in the $\boldsymbol{k}$ direction,

$$
\boldsymbol{B}_{0}=\left(0, B_{1}, B_{2}\right)^{T}
$$

where the desired component of the field $B_{1} \gg B_{2}$. Introducing (3) and (4) into (2), the differential of the Lorentz force is found to be

$$
\mathrm{d} \boldsymbol{F}_{L}=I\left(\frac{\partial Y}{\partial s} B_{2}-\frac{\partial Z}{\partial s} B_{1},-\frac{\partial X}{\partial s} B_{2}, \frac{\partial X}{\partial s} B_{1}\right)^{T} \mathrm{~d} s .
$$

The term $-\frac{\partial Z}{\partial s} B_{1} I d s$ in the $\boldsymbol{i}$ component opposes the motion and drags the ET system, as intended. However, the crossed $B_{2}$ terms in the $\boldsymbol{i}$ and $\boldsymbol{j}$ components (due to the imperfection $B_{2}$ ) will tend to coil the tether. This undesirable effect has been reported in some tether flights [2, 11].

In this paper we study the interaction of elastic and electromagnetic forces in a conducting rod and the tendency to generate three-dimensional coiled configurations (for instance as a result of buckling at critical loads).

\section{THE ROD MECHANICS MODEL}

We describe the elastic behaviour of a conducting cable by the Kirchhoff equations for the dynamics of thin rods. The rod is assumed to be uniform, inextensible, unshearable 


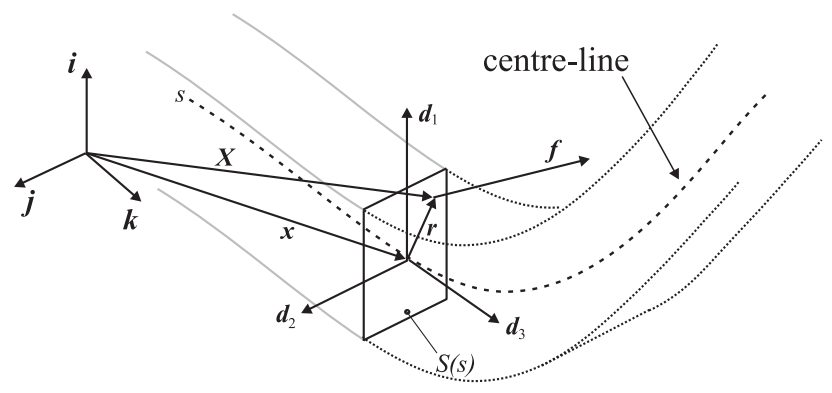

FIG. 3: Cosserat model of a rod.

and intrinsically straight and prismatic. The inextensible and unshearable assumptions are appropriate for thin rods with low external forces (electrodynamic), as the tether. For the background of the Kirchhoff equations the reader is referred to [1, 3]. These equations were also used in [19] to analyse the dynamics of the SET. To these equations will be added the Lorentz force to account for the electromagnetic interaction.

Let $\boldsymbol{x}$ denote the position of the rod's centreline and let $\left\{\boldsymbol{d}_{1}, \boldsymbol{d}_{2}, \boldsymbol{d}_{3}\right\}$ be a right-handed orthonormal frame of directors (the Cosserat triad) defined at each point along the centreline. Since the centreline is assumed to be inextensible we can take $\boldsymbol{d}_{3}$ in the direction of the local tangent:

$$
\boldsymbol{x}^{\prime}(s, t)=\boldsymbol{d}_{3}(s, t),
$$

where the prime denotes differentiation with respect to arclength $s$ measured along the centreline, and $t$ is time. The directors $\boldsymbol{d}_{1}$ and $\boldsymbol{d}_{2}$ will be taken to point along the principal bending axes of the cross-section (see Figure 3). The unstressed rod is taken to lie along the basis vector $\boldsymbol{k}$ of a fixed inertial frame $\{\boldsymbol{i}, \boldsymbol{j}, \boldsymbol{k}\}$.

Looking at Figure 3 we note that the position vector of an arbitrary point of the rod can be expressed as

$$
\begin{aligned}
\boldsymbol{X}\left(s, \xi_{1}, \xi_{2}, t\right) & =\boldsymbol{x}(s, t)+\xi_{1} \boldsymbol{d}_{1}(s, t)+\xi_{2} \boldsymbol{d}_{2}(s, t) \\
& =\boldsymbol{x}(s, t)+\boldsymbol{r}\left(s, \xi_{1}, \xi_{2}, t\right),
\end{aligned}
$$

where $\left(\xi_{1}, \xi_{2}\right)$ are the components of $\boldsymbol{r}$ in the cross-section relative to $\left\{\boldsymbol{d}_{1}(s), \boldsymbol{d}_{2}(s)\right\}$. The rod is thus viewed as a set of infinitesimal slices centred at all $s$. A one-dimensional description will be obtained by averaging of forces and moments over each cross-section. The internal traction, which is the projection of the stress tensor onto the cross-sectional plane, is given by a force which we denote by $\boldsymbol{f}=\boldsymbol{f}\left(s, \xi_{1}, \xi_{2}, t\right)$ (see Figure 3). The resultant elastic force 
exerted in a section $S(s)$ is given by

$$
\boldsymbol{F}(s, t)=\int_{S(s)} \boldsymbol{f}\left(s, \xi_{1}, \xi_{2}, t\right) \mathrm{d} S
$$

where $\mathrm{d} S$ is an infinitesimal area element. This force can be expressed in the director basis as $\boldsymbol{F}=\sum_{i=1}^{3} F_{i} \boldsymbol{d}_{i}$. The resultant moment in the section $S(s)$ is given by

$$
\boldsymbol{M}(s, t)=\int_{S(s)} \boldsymbol{r}\left(s, \xi_{1}, \xi_{2}, t\right) \times \boldsymbol{f}\left(s, \xi_{1}, \xi_{2}, t\right) \mathrm{d} S
$$

and will be expressed as $\boldsymbol{M}=\sum_{i=1}^{3} M_{i} \boldsymbol{d}_{i}$.

The rod is assumed to carry an electric current for which we can write

$$
\boldsymbol{I}=I \boldsymbol{x}^{\prime}=I \boldsymbol{d}_{3}
$$

Here we have assumed the current to have the same direction as the rod, which is consistent with a one-dimensional rod theory. It amounts to the assumption that the cross-section of the conducting wire is small enough to make currents within the cross-section (eddy currents) induced by the motion negligible. The current $\boldsymbol{I}$ interacts with the magnetic field $\boldsymbol{B}_{0}$ to generate a (Lorentz) body force given by

$$
\boldsymbol{F}_{L}=I \boldsymbol{d}_{3} \times \boldsymbol{B}_{0}
$$

Following [21] we assume the magnetic field to be uniform and directed along the unstressed rod, i.e.,

$$
\boldsymbol{B}_{0}=B_{0} \boldsymbol{k},
$$

modelling the undesired component of the magnetic field, $B_{2}$ in equation (4).

The balancing of forces and moments across an infinitesimal rod element [19] then yields the following set of partial differential equations:

$$
\begin{gathered}
\boldsymbol{F}^{\prime}+I B_{0} \boldsymbol{d}_{3} \times \boldsymbol{k}=\rho A \ddot{\boldsymbol{x}}, \\
\boldsymbol{M}^{\prime}+\boldsymbol{d}_{3} \times \boldsymbol{F}=\rho\left(I_{2} \boldsymbol{d}_{1} \times \ddot{\boldsymbol{d}}_{1}+I_{1} \boldsymbol{d}_{2} \times \ddot{\boldsymbol{d}}_{2}\right),
\end{gathered}
$$

where $\rho$ is the (volumetric) mass density, $A$ the cross-sectional area, $I_{1}$ and $I_{2}$ the second moment of area of the cross-section about $\boldsymbol{d}_{1}$ and $\boldsymbol{d}_{2}$ respectively, and ( ) denotes differentiation with respect to time. 
For a closed system of equations these balance equations need to be supplemented by constitutive relations that characterise the material behaviour of the rod. We assume the rod to be made of homogeneous isotropic linear viscoelastic material so that stress-strain relations, based on a model by Valverde et al. [19], are

$$
\begin{aligned}
& M_{1}=E I_{1}\left(\kappa_{1}+\gamma_{v} \dot{\kappa}_{1}\right), \\
& M_{2}=E I_{2}\left(\kappa_{2}+\gamma_{v} \dot{\kappa}_{2}\right), \\
& M_{3}=G J\left(\kappa_{3}+\gamma_{v} \dot{\kappa}_{3}\right),
\end{aligned}
$$

where $\kappa_{1}$ and $\kappa_{2}$ are the curvatures about $\boldsymbol{d}_{1}$ and $\boldsymbol{d}_{2}$, respectively, while $\kappa_{3}$ is the twist about $\boldsymbol{d}_{3}$. The constant $\gamma_{v}$ is the viscoelastic coefficient of the material, $E$ is Young's modulus, $G$ is the shear modulus and $J$ is the second moment of area of the cross-section about $\boldsymbol{d}_{3}$. We shall assume that the section is symmetric with respect to the principal axes, in which case $J=I_{1}+I_{2}$.

The $\kappa_{i}$ are the components of the curvature vector

$$
\boldsymbol{\kappa}=\sum_{i=1}^{3} \kappa_{i} \boldsymbol{d}_{i}
$$

which governs the evolution in space of the frame of directors as one moves along the centreline:

$$
\boldsymbol{d}_{i}^{\prime}=\boldsymbol{\kappa} \times \boldsymbol{d}_{i} \quad(i=1,2,3) .
$$

The constitutive relations (15) can be used to replace the $\kappa_{i}$ in (17) by moments, after which the equations (6), (13), (14) and (17) form a system of 18 differential equations for the 18 unknowns $\left(\boldsymbol{x}, \boldsymbol{F}, \boldsymbol{M}, \boldsymbol{d}_{1}, \boldsymbol{d}_{2}, \boldsymbol{d}_{3}\right)$.

Remark: In general when a conducting wire moves in a magnetic field an additional electromagnetic induction effect occurs which opposes the motion. The electromotive force as

a result of this effect is proportional to the rate of change of the enclosed magnetic flux [9]. However, in the case of a steadily whirling wire in a uniform magnetic field the enclosed magnetic flux does not change and the effect is zero.

\section{A. Equations of motion in the moving frame}

Since we are interested in steadily rotating solutions we transform the equilibrium equations (13) and (14) to a co-ordinate frame $\left\{\boldsymbol{e}_{1}, \boldsymbol{e}_{2}, \boldsymbol{e}_{3}\right\}$ that rotates with constant angular 
velocity $\boldsymbol{\omega}=\omega \boldsymbol{k}$ about the $\boldsymbol{k}$ axis (and the axis of the rod in its trivial unstressed state). Noting that the derivative with respect to time of an arbitrary vector $\boldsymbol{V}(s, t)$ is given by

$$
\left.\frac{d \boldsymbol{V}(s, t)}{d t}\right|_{i}=\left.\frac{d \boldsymbol{V}(s, t)}{d t}\right|_{m}+\boldsymbol{\omega} \times \boldsymbol{V}(s, t),
$$

where $\left.\frac{d}{d t}\right|_{i}$ indicates the derivative with respect to time in the inertial frame and $\left.\frac{d}{d t}\right|_{m}$ stands for the derivative with respect to time in the moving frame, the equations (13) and (14) expressed relative to $\left\{\boldsymbol{e}_{1}, \boldsymbol{e}_{2}, \boldsymbol{e}_{3}\right\}$ become

$$
\begin{gathered}
\boldsymbol{F}^{\prime}+I B_{0} \boldsymbol{d}_{3} \times \boldsymbol{e}_{3}=\rho A(\ddot{\boldsymbol{x}}+2 \boldsymbol{\omega} \times \dot{\boldsymbol{x}}+\boldsymbol{\omega} \times(\boldsymbol{\omega} \times \boldsymbol{x})) \\
\boldsymbol{M}^{\prime}+\boldsymbol{d}_{3} \times \boldsymbol{F} \\
=\rho I_{2}\left(\boldsymbol{d}_{1} \times \ddot{\boldsymbol{d}}_{1}+2 \boldsymbol{d}_{1} \times\left(\boldsymbol{\omega} \times \dot{\boldsymbol{d}}_{1}\right)+\left(\boldsymbol{\omega} \cdot \boldsymbol{d}_{1}\right)\left(\boldsymbol{d}_{1} \times \boldsymbol{\omega}\right)\right) \\
+\rho I_{1}\left(\boldsymbol{d}_{2} \times \ddot{\boldsymbol{d}}_{2}+2 \boldsymbol{d}_{2} \times\left(\boldsymbol{\omega} \times \dot{\boldsymbol{d}}_{2}\right)+\left(\boldsymbol{\omega} \cdot \boldsymbol{d}_{2}\right)\left(\boldsymbol{d}_{2} \times \boldsymbol{\omega}\right)\right) .
\end{gathered}
$$

Steadily rotating (whirling) solutions satisfy the equations (19) and (20) with the dotted variables set to zero:

$$
\begin{gathered}
\boldsymbol{F}^{\prime}+I B_{0} \boldsymbol{d}_{3} \times \boldsymbol{e}_{3}=\rho A \boldsymbol{\omega} \times(\boldsymbol{\omega} \times \boldsymbol{x}) \\
\boldsymbol{M}^{\prime}+\boldsymbol{d}_{3} \times \boldsymbol{F}=\rho I_{2}\left(\boldsymbol{\omega} \cdot \boldsymbol{d}_{1}\right)\left(\boldsymbol{d}_{1} \times \boldsymbol{\omega}\right)+\rho I_{1}\left(\boldsymbol{\omega} \cdot \boldsymbol{d}_{2}\right)\left(\boldsymbol{d}_{2} \times \boldsymbol{\omega}\right) .
\end{gathered}
$$

The other equations (6) and (17) do not change their form, but all vectors are now to be considered as expressed relative to the rotating frame $\left\{\boldsymbol{e}_{1}, \boldsymbol{e}_{2}, \boldsymbol{e}_{3}\right\}$. Statical solutions are simply obtained by setting $\omega$ equal to zero.

For a well-posed problem the final 18 ODEs require 18 boundary conditions to be specified.

\section{B. The boundary conditions}

We follow [23] and consider welded boundary conditions. These conditions also describe an electrodynamic tether that is welded to the end contactors or modules if these bodies are sufficiently massive (see Figure 2). We assume the rod to be fixed at $s=L$ and to be able to slide along $\boldsymbol{e}_{3}=\boldsymbol{k}$ at $s=0$ where a controlled force $T$ is applied (positive for tension). $L$ is the length of the rod. Writing $\boldsymbol{x}=x \boldsymbol{e}_{1}+y \boldsymbol{e}_{2}+z \boldsymbol{e}_{3}$ a consistent set of boundary conditions 
is thus given by

$$
\begin{array}{r}
x(0, t)=0, \\
y(0, t)=0, \\
\boldsymbol{F}(0, t) \cdot \boldsymbol{e}_{3}=T, \\
\boldsymbol{d}_{3}(0, t) \cdot \boldsymbol{e}_{1}=0, \\
\boldsymbol{d}_{3}(0, t) \cdot \boldsymbol{e}_{2}=0, \\
\boldsymbol{d}_{1}(0, t) \cdot \boldsymbol{e}_{2}=0,
\end{array}
$$

at $s=0$ and

$$
\begin{aligned}
x(L, t) & =0, \\
y(L, t) & =0, \\
z(L, t) & =L, \\
\boldsymbol{d}_{3}(L, t) \cdot \boldsymbol{e}_{1} & =0, \\
\boldsymbol{d}_{3}(L, t) \cdot \boldsymbol{e}_{2} & =0, \\
\boldsymbol{d}_{1}(L, t) \cdot \boldsymbol{e}_{2} & =0,
\end{aligned}
$$

at $s=L$. To these conditions we have to add conditions that ensure the orthonormality of the director basis, for which we can take

$$
\begin{aligned}
& \boldsymbol{d}_{1}(0, t) \cdot \boldsymbol{d}_{1}(0, t)=1 \\
& \boldsymbol{d}_{2}(0, t) \cdot \boldsymbol{d}_{2}(0, t)=1 \\
& \boldsymbol{d}_{3}(0, t) \cdot \boldsymbol{d}_{3}(0, t)=1 \\
& \boldsymbol{d}_{1}(0, t) \cdot \boldsymbol{d}_{2}(0, t)=0 \\
& \boldsymbol{d}_{1}(0, t) \cdot \boldsymbol{d}_{3}(0, t)=0 \\
& \boldsymbol{d}_{2}(0, t) \cdot \boldsymbol{d}_{3}(0, t)=0
\end{aligned}
$$

for a total of 18 boundary conditions, as required. Note that these conditions imply that at $s=0$ and $s=L$ the director frame $\left\{\boldsymbol{d}_{1}, \boldsymbol{d}_{2}, \boldsymbol{d}_{3}\right\}$ is aligned with $\left\{\boldsymbol{e}_{1}, \boldsymbol{e}_{2}, \boldsymbol{e}_{3}\right\}$. 


\section{NONDIMENSIONALISATION}

We make the system of equations dimensionless by scaling the variables in the following way

$$
\begin{aligned}
& \omega_{c}=\sqrt{f \frac{E I_{1}}{\rho A L^{4}}}, \quad \bar{t}=t \omega_{c}, \quad \bar{s}=\frac{s}{L} \in[0,1], \quad \bar{\omega}=\frac{\omega}{\omega_{c}}, \quad \overline{\boldsymbol{x}}=\frac{\boldsymbol{x}}{L}, \\
& \overline{\boldsymbol{F}}=\boldsymbol{F} \frac{L^{2}}{f E I_{1}}, \quad \bar{T}=T \frac{L^{2}}{f E I_{1}}, \quad \overline{\boldsymbol{M}}=\boldsymbol{M} \frac{L}{f E I_{1}}, \quad \overline{\boldsymbol{\kappa}}=\boldsymbol{\kappa} L .
\end{aligned}
$$

Here $\omega_{c}$ is a reference bending natural frequency which, through tuning of the numerical constant $f$, can be adapted to the particular boundary conditions at hand and the natural mode considered. In the analysis in Section VI we shall take $f=1$, but since in the welded case we consider realistic data for electrodynamic tethers we shall take $f=500.5639$ in that case so as to get convenient numbers when presenting our numerical results. This value for $f$ corresponds to the first bending natural frequency of a welded-welded beam about $\boldsymbol{d}_{1}$.

With this nondimensionalisation the equations become (dropping the overbars for simplicity and letting a prime denote $\left.\frac{d}{d \bar{s}}\right)$ :

$$
\begin{gathered}
\boldsymbol{F}^{\prime}+B \boldsymbol{d}_{3} \times \boldsymbol{k}=\ddot{\boldsymbol{x}}+2 \boldsymbol{\omega} \times \dot{\boldsymbol{x}}+\boldsymbol{\omega} \times(\boldsymbol{\omega} \times \boldsymbol{x}), \\
\boldsymbol{M}^{\prime}+\boldsymbol{d}_{3} \times \boldsymbol{F}=P\left[R\left(\boldsymbol{d}_{1} \times \ddot{\boldsymbol{d}}_{1}+2 \boldsymbol{d}_{1} \times \boldsymbol{\omega} \times \dot{\boldsymbol{d}}_{1}+\left(\boldsymbol{\omega} \cdot \boldsymbol{d}_{1}\right)\left(\boldsymbol{d}_{1} \times \boldsymbol{\omega}\right)\right)\right. \\
\left.+\left(\boldsymbol{d}_{2} \times \ddot{\boldsymbol{d}}_{2}+2 \boldsymbol{d}_{2} \times \boldsymbol{\omega} \times \dot{\boldsymbol{d}}_{2}+\left(\boldsymbol{\omega} \cdot \boldsymbol{d}_{2}\right)\left(\boldsymbol{d}_{2} \times \boldsymbol{\omega}\right)\right)\right] \\
\boldsymbol{x}^{\prime}=\boldsymbol{d}_{3}, \\
\boldsymbol{d}_{i}^{\prime}=\boldsymbol{\kappa} \times \boldsymbol{d}_{i}
\end{gathered}
$$

and the constitutive relations can be written as

$$
\boldsymbol{M}=\frac{1}{f}\left[\left(\kappa_{1}+\gamma \dot{\kappa_{1}}\right) \boldsymbol{d}_{1}+R\left(\kappa_{2}+\gamma \dot{\kappa_{2}}\right) \boldsymbol{d}_{2}+\frac{\Gamma(1+R)}{2}\left(\kappa_{3}+\gamma \dot{\kappa_{3}}\right) \boldsymbol{d}_{3}\right],
$$

where the dimensionless parameters are

$$
P=\frac{I_{1}}{A L^{2}}, \quad R=\frac{I_{2}}{I_{1}}, \quad B=\frac{B_{0} I L^{3}}{f E I_{1}}, \quad \Gamma=\frac{2 G}{E}, \quad \gamma=\gamma_{v} \omega_{c},
$$

and $\left(\frac{1}{\Gamma}-1\right)$ is equal to Poisson's ratio. For the boundary conditions we can still use (23) to (35) if we assume that they now refer to dimensionless variables and that the right-hand conditions are imposed at $\bar{s}=1$. 


\section{PERTURBATION SCHEME}

We consider whirling solutions (relative equilibria) that are stationary in the moving frame $\left\{\boldsymbol{e}_{1}, \boldsymbol{e}_{2}, \boldsymbol{e}_{3}\right\}$. Such solutions are found by solving the set of equations (37)-(41) with the dotted variables set to zero (thus obtaining an ODE). To study their stability we linearise the full PDE (37)-(41) about these whirling solutions. We follow the approach in [19], which is similar to approaches in [5, 6]. The stability of statical (non-whirling) solutions can be investigated by simply setting the angular velocity $\omega$ to zero.

We start our perturbation analysis by writing

$$
\boldsymbol{d}_{i}(s, t)=\boldsymbol{d}_{i}^{0}(s)+\delta \boldsymbol{d}_{i}^{t}(s, t)+O\left(\delta^{2}\right), \quad i=1,2,3,
$$

where $\boldsymbol{d}_{i}^{0}(s)$ are the basis vectors of a quasi-stationary solution, $\boldsymbol{d}_{i}^{t}(s, t)$ are the basis vectors of a time-dependent perturbation and $\delta$ is a small bookkeeping parameter introduced to separate scales. Note that, in order to preserve orthonormality to $O(\delta)\left(\boldsymbol{d}_{i} \cdot \boldsymbol{d}_{j}=\delta_{i j}+O\left(\delta^{2}\right)\right)$, we must have

$$
\boldsymbol{d}_{i}^{t}(s, t)=\sum_{j=1}^{3} A_{i j}(s, t) \boldsymbol{d}_{j}^{0}(s), \quad i=1,2,3,
$$

where the matrix $A_{i j}$ is skew-symmetric and can be written as

$$
\boldsymbol{A}=\left(\begin{array}{ccc}
0 & \alpha_{3} & -\alpha_{2} \\
-\alpha_{3} & 0 & \alpha_{1} \\
\alpha_{2} & -\alpha_{1} & 0
\end{array}\right)
$$

Thus, the nine components of the director basis perturbation are described by only three independent parameters, and if we introduce

$$
\boldsymbol{\alpha}=\left(\alpha_{1}, \alpha_{2}, \alpha_{3}\right)^{T}
$$

(with respect to the unperturbed director basis) then the perturbed director basis can be expressed as

$$
\boldsymbol{d}_{i}(s, t)=\boldsymbol{d}_{i}^{0}(s)+\delta \boldsymbol{\alpha}(s, t) \times \boldsymbol{d}_{i}^{0}(s)+O\left(\delta^{2}\right), \quad i=1,2,3 .
$$

Using (47), the perturbation of an arbitrary vector $\boldsymbol{V}=\sum_{i=1}^{3} V_{i} \boldsymbol{d}_{i}$ can be written on the basis $\left\{\boldsymbol{d}_{1}^{0}, \boldsymbol{d}_{2}^{0}, \boldsymbol{d}_{3}^{0}\right\}$ as

$$
\boldsymbol{V}=\boldsymbol{V}^{0}+\delta \boldsymbol{V}^{t}+O\left(\delta^{2}\right)=\sum_{i=1}^{3}\left[V_{i}^{0}+\delta\left(V_{i}^{t}+\left(\boldsymbol{\alpha} \times \boldsymbol{V}^{0}\right)_{i}\right)\right] \boldsymbol{d}_{i}^{0}+O\left(\delta^{2}\right),
$$


where ()$_{i}$ denotes the component along $\boldsymbol{d}_{i}^{0}$ and time and space dependence of the variables have been suppressed for the sake of simplicity.

Applying this perturbation scheme to the PDEs (37)-(41) and the boundary conditions, we arrive at an $O(1)$ nonlinear ODE for the quasi-stationary solutions and an $O(\delta)$ linear PDE governing their stability.

\section{A. The $O(1)$ equations - quasi-stationary whirl}

The $O(1)$ equations are time-independent. Recalling that $\boldsymbol{\omega}=\boldsymbol{\omega} \boldsymbol{e}_{3}$, we find the $O(1)$ terms of the linear momentum equation (37), projected on the director basis $\left\{\boldsymbol{d}_{1}^{0}, \boldsymbol{d}_{2}^{0}, \boldsymbol{d}_{3}^{0}\right\}$, to give

$$
\begin{aligned}
\left(F_{1}^{0}\right)^{\prime}-F_{2}^{0} \kappa_{3}^{0}+F_{3}^{0} \kappa_{2}^{0}+B\left(d_{32}^{0} d_{11}^{0}-d_{31}^{0} d_{12}^{0}\right) & =-\omega^{2}\left(x^{0} d_{11}^{0}+y^{0} d_{12}^{0}\right), \\
\left(F_{2}^{0}\right)^{\prime}-F_{3}^{0} \kappa_{1}^{0}+F_{1}^{0} \kappa_{3}^{0}+B\left(d_{32}^{0} d_{21}^{0}-d_{31}^{0} d_{22}^{0}\right) & =-\omega^{2}\left(x^{0} d_{21}^{0}+y^{0} d_{22}^{0}\right), \\
\left(F_{3}^{0}\right)^{\prime}-F_{1}^{0} \kappa_{2}^{0}+F_{2}^{0} \kappa_{1}^{0} & =-\omega^{2}\left(x^{0} d_{31}^{0}+y^{0} d_{32}^{0}\right),
\end{aligned}
$$

where subscripts are used to indicate components relative to the basis vectors $\left\{\boldsymbol{d}_{1}^{0}, \boldsymbol{d}_{2}^{0}, \boldsymbol{d}_{3}^{0}\right\}$ (but the $\boldsymbol{d}_{i}^{0}$ components are relative to $\left\{\boldsymbol{e}_{1}, \boldsymbol{e}_{2}, \boldsymbol{e}_{3}\right\}$ ). Similarly, the $O(1)$ term of the angular momentum equation (38), projected on the director basis $\left\{\boldsymbol{d}_{1}^{0}, \boldsymbol{d}_{2}^{0}, \boldsymbol{d}_{3}^{0}\right\}$ gives

$$
\begin{aligned}
& \left(M_{1}^{0}\right)^{\prime}=\frac{2 f M_{3}^{0} M_{2}^{0}}{\Gamma(1+R)}-\frac{f M_{2}^{0} M_{3}^{0}}{R}+F_{2}^{0}+P \omega^{2} d_{23}^{0}\left(d_{22}^{0} d_{11}^{0}-d_{21}^{0} d_{12}^{0}\right) \\
& \left(M_{2}^{0}\right)^{\prime}=-\frac{2 f M_{3}^{0} M_{1}^{0}}{\Gamma(1+R)}+f M_{1}^{0} M_{3}^{0}-F_{1}^{0}+P R \omega^{2} d_{13}^{0}\left(d_{21}^{0} d_{12}^{0}-d_{11}^{0} d_{22}^{0}\right) \\
& \left(M_{3}^{0}\right)^{\prime}=\frac{f M_{2}^{0} M_{1}^{0}}{R}-f M_{1}^{0} M_{2}^{0}+P R \omega^{2} d_{13}^{0}\left(d_{12}^{0} d_{31}^{0}-d_{11}^{0} d_{32}^{0}\right)+P \omega^{2} d_{23}^{0}\left(d_{22}^{0} d_{31}^{0}-d_{21}^{0} d_{32}^{0}\right)
\end{aligned}
$$

The $O(1)$ term of equation (39) can be expressed as

$$
\left(\boldsymbol{x}^{0}\right)^{\prime}=\boldsymbol{d}_{3}^{0},
$$

and the twist equation (40) by

$$
\left(\boldsymbol{d}_{i}^{0}\right)^{\prime}=\boldsymbol{\kappa}^{0} \times \boldsymbol{d}_{i}^{0}, \quad i=1,2,3
$$


where $\boldsymbol{\kappa}^{0}=\sum_{j=1}^{3} \kappa_{j}^{0} \boldsymbol{d}_{j}^{0}$. The $O(1)$ term of constitutive relations (41) can be expressed as

$$
M_{1}^{0}=\frac{1}{f} \kappa_{1}^{0}, \quad M_{2}^{0}=\frac{R}{f} \kappa_{2}^{0}, \quad M_{1}^{0}=\frac{\Gamma(1+R)}{2 f} \kappa_{3}^{0},
$$

which can be used to express the $\kappa_{i}^{0}$ in (56) in terms of the moments $M_{i}^{0}$.

Proceeding in the same way, the $O(1)$ part of the boundary conditions is given by

$$
\begin{array}{r}
x^{0}(0)=0, \\
y^{0}(0)=0, \\
\boldsymbol{F}^{0}(0) \cdot \boldsymbol{e}_{3}=T, \\
\boldsymbol{d}_{3}^{0}(0) \cdot \boldsymbol{e}_{1}=0, \\
\boldsymbol{d}_{3}^{0}(0) \cdot \boldsymbol{e}_{2}=0, \\
\boldsymbol{d}_{1}^{0}(0) \cdot \boldsymbol{e}_{2}=0,
\end{array}
$$

$$
\begin{gathered}
x^{0}(1)=0, \\
y^{0}(1)=0, \\
z^{0}(1)=1, \\
\boldsymbol{d}_{3}^{0}(1) \cdot \boldsymbol{e}_{1}=0, \\
\boldsymbol{d}_{3}^{0}(1) \cdot \boldsymbol{e}_{2}=0, \\
\boldsymbol{d}_{1}^{0}(1) \cdot \boldsymbol{e}_{2}=0, \\
\boldsymbol{d}_{1}^{0}(0) \cdot \boldsymbol{d}_{1}^{0}(0)=1, \\
\boldsymbol{d}_{2}^{0}(0) \cdot \boldsymbol{d}_{2}^{0}(0)=1, \\
\boldsymbol{d}_{3}^{0}(0) \cdot \boldsymbol{d}_{3}^{0}(0)=1, \\
\boldsymbol{d}_{1}^{0}(0) \cdot \boldsymbol{d}_{2}^{0}(0)=0, \\
\boldsymbol{d}_{1}^{0}(0) \cdot \boldsymbol{d}_{3}^{0}(0)=0, \\
\boldsymbol{d}_{2}^{0}(0) \cdot \boldsymbol{d}_{3}^{0}(0)=0,
\end{gathered}
$$




\section{B. The $O(\delta)$ equations - linearisation}

The $O(\delta)$ part of the linear momentum equation (37) can be written as

$$
\begin{aligned}
\left(\boldsymbol{F}^{t}(s, t)\right)^{\prime} & +\boldsymbol{B}_{1}(s) \boldsymbol{F}^{t}(s, t)+\boldsymbol{B}_{2}(s) \boldsymbol{x}^{t}(s, t)+\boldsymbol{B}_{3}(s) \boldsymbol{\alpha}^{\prime}(s, t)+\boldsymbol{B}_{4}(s) \boldsymbol{\alpha}(s, t) \\
& =\boldsymbol{B}_{5}(s) \ddot{\boldsymbol{x}}^{t}(s, t)+\boldsymbol{B}_{6}(s) \dot{\boldsymbol{x}}^{t}(s, t)
\end{aligned}
$$

where the $3 \times 3$ matrices $\boldsymbol{B}_{i}(s)$ are given in the Appendix. Here we have expressed $\boldsymbol{F}^{t}$ relative to $\left\{\boldsymbol{d}_{1}^{0}, \boldsymbol{d}_{2}^{0}, \boldsymbol{d}_{3}^{0}\right\}$ and $\boldsymbol{x}^{t}$ relative to $\left\{\boldsymbol{e}_{1}, \boldsymbol{e}_{2}, \boldsymbol{e}_{3}\right\}$. For the $O(\delta)$ part of the angular momentum equation (38) we can write

$$
\begin{aligned}
\left(\boldsymbol{M}^{t}(s, t)\right)^{\prime} & +\boldsymbol{C}_{1}(s) \boldsymbol{M}^{t}(s, t)+\boldsymbol{C}_{2}(s) \boldsymbol{\alpha}^{\prime}(s, t)+\boldsymbol{C}_{3}(s) \boldsymbol{\alpha}(s, t)+\boldsymbol{C}_{4}(s) \boldsymbol{F}^{t}(s, t) \\
& =\boldsymbol{C}_{5}(s) \ddot{\boldsymbol{\alpha}}(s, t)+\boldsymbol{C}_{6}(s) \dot{\boldsymbol{\alpha}}(s, t),
\end{aligned}
$$

where the matrices $\boldsymbol{C}_{i}(s)$ are again given in the Appendix. $\boldsymbol{M}^{t}$ is expressed relative to $\left\{\boldsymbol{d}_{1}^{0}, \boldsymbol{d}_{2}^{0}, \boldsymbol{d}_{3}^{0}\right\}$. The 9 twist equations (40) at $O(\delta)$ are reduced to only 3 independent equations that relate $\boldsymbol{\kappa}^{t}$ and $\boldsymbol{\alpha}$ as

$$
\boldsymbol{\kappa}^{t}(s, t)=\boldsymbol{\alpha}^{\prime}(s, t)+\boldsymbol{\kappa}^{0}(s) \times \boldsymbol{\alpha}(s, t) .
$$

Introducing these relations into the $O(\delta)$ part of the constitutive relations gives

$$
\boldsymbol{M}^{t}(s, t)+\boldsymbol{D}_{1}(s) \boldsymbol{\alpha}^{\prime}(s, t)+\boldsymbol{D}_{2}(s) \boldsymbol{\alpha}(s, t)=\boldsymbol{D}_{3}(s) \dot{\boldsymbol{\alpha}}(s, t)+\boldsymbol{D}_{4}(s) \frac{\partial^{2}}{\partial s \partial t}(\boldsymbol{\alpha}(s, t)),
$$

where the matrices $\boldsymbol{D}_{i}(s)$ are given in the Appendix. Finally, the $O(\delta)$ part of equation (39) yields

$$
\left(\boldsymbol{x}^{t}(s, t)\right)^{\prime}=\alpha(s, t) \times \boldsymbol{d}_{3}^{0}(s) .
$$

Applying the perturbation scheme to the boundary conditions at $O(\delta)$, we obtain

$$
\begin{aligned}
x^{t}(0, t) & =0, \\
y^{t}(0, t) & =0, \\
\boldsymbol{F}^{t}(0, t) \cdot \boldsymbol{e}_{3} & =0, \\
\boldsymbol{\alpha}(0, t) & =\mathbf{0}, \\
\boldsymbol{x}^{t}(1, t) & =\mathbf{0}, \\
\boldsymbol{\alpha}(1, t) & =\mathbf{0} .
\end{aligned}
$$


After elimination of the $\kappa_{i}^{0}$ by means of (157), the set of 12 equations (71), (172), (74) and (75) together with the 12 boundary conditions (76)-(81), with appropriate initial conditions, form a well-posed initial-boundary-value problem.

\section{STABILITY ANALYSIS}

Since we are interested in stability of solutions we look for solutions of the $O(\delta)$ equations of the form

$$
\begin{aligned}
\boldsymbol{x}^{t}(s, t) & =\hat{\boldsymbol{x}}^{t}(s) e^{\lambda t}, \\
\boldsymbol{\alpha}(s, t) & =\hat{\boldsymbol{\alpha}}(s) e^{\lambda t}, \\
\boldsymbol{F}^{t}(s, t) & =\hat{\boldsymbol{F}}^{t}(s) e^{\lambda t}, \\
\boldsymbol{M}^{t}(s, t) & =\hat{\boldsymbol{M}}^{t}(s) e^{\lambda t} .
\end{aligned}
$$

When these expressions are inserted into (71)-(75) a linear eigenvalue problem for a 12 dimensional ODE is obtained in terms of the variables $\left(\hat{\boldsymbol{x}}^{t}, \hat{\boldsymbol{\alpha}}^{t}, \hat{\boldsymbol{F}}^{t}, \hat{\boldsymbol{M}}^{t}\right)$. The eigenvalue $\lambda$ measures the growth of small perturbations and is to be found as past of the solution. Eigenvalues come as complex conjugate pairs. A whirling state is unstable if at least one of the (in general infinitely many) $\lambda$ 's has positive real part.

To solve a real system of equations we split the eigenvalues and variables (eigenfunctions) into real and imaginary parts, $\lambda=\lambda_{r}+i \lambda_{i}, \hat{\boldsymbol{x}}^{t}=\hat{\boldsymbol{x}}_{r}^{t}+i \hat{\boldsymbol{x}}_{i}^{t}, \hat{\boldsymbol{\alpha}}^{t}=\hat{\boldsymbol{\alpha}}_{r}^{t}+i \hat{\boldsymbol{\alpha}}_{i}^{t}, \hat{\boldsymbol{F}}^{t}=\hat{\boldsymbol{F}}_{r}^{t}+i \hat{\boldsymbol{F}}_{i}^{t}$ and $\hat{\boldsymbol{M}}^{t}=\hat{\boldsymbol{M}}_{r}^{t}+i \hat{\boldsymbol{M}}_{i}^{t}$. The equations (71) -(75), along with the boundary conditions (76)(81), are similarly split into real and imaginary parts. Thus we end up with a doubled 24-dimensional linearised boundary-value problem.

\section{A. Stability of the straight rod - magnetic buckling}

The trivial solution of the $O(1)$ equations (49)-(57), representing a straight and untwisted rod, is given by

$$
\boldsymbol{x}(s)=s \boldsymbol{e}_{3}, \quad \boldsymbol{F}(s)=-T \boldsymbol{e}_{3}, \quad \boldsymbol{M}(s)=\mathbf{0}, \quad \boldsymbol{d}_{i}(s)=\boldsymbol{e}_{i} \quad(i=1,2,3), \quad s \in[0,1] .
$$


For the statical case $(\omega=0)$ without end force $(T=0)$ the $O(\delta)$ equations (71), (72), (74)), (75) about this trivial solution, on inserting (82)-(85), can be written as

$$
\begin{aligned}
& x^{\prime \prime \prime \prime}-\lambda^{2} P x^{\prime \prime}+\frac{\lambda^{2}}{R} x-\frac{B}{R} y^{\prime}=0, \\
& y^{\prime \prime \prime \prime}-\lambda^{2} P y^{\prime \prime}+\lambda^{2} y+B x^{\prime}=0, \\
& M_{3}^{\prime \prime}-\frac{2 \lambda^{2} P}{\Gamma} M_{3}=0,
\end{aligned}
$$

with boundary conditions

$$
x(0)=x(1)=x^{\prime}(0)=x^{\prime}(1)=y(0)=y(1)=y^{\prime}(0)=y^{\prime}(1)=M_{3}^{\prime}(0)=M_{3}^{\prime}(1)=0,
$$

while $F_{3} \equiv 0, z \equiv 0$. Note that the torsional $\left(M_{3}\right)$ modes decouple from the bending $(x, y)$ modes.

To find the statical magnetic buckling loads we set $\lambda=0$. The bending equations then reduce to

$$
z^{\prime \prime \prime \prime \prime \prime \prime \prime ~}+\frac{B^{2}}{R} z=0, \quad \text { for } \quad z=x^{\prime}
$$

subject to

$$
x(0)=x(1)=x^{\prime}(0)=x^{\prime}(1)=x^{\prime \prime \prime \prime}(0)=x^{\prime \prime \prime \prime}(1)=0 .
$$

On setting $z=e^{i k s}$ we obtain the characteristic equation $-k^{6}+B^{2} / R=0$ with solutions $k_{1,2}= \pm \beta, k_{3,4,5,6}= \pm \beta(1 \pm i \sqrt{3}) / 2$, where $\beta=B^{1 / 3} / R^{1 / 6}$. Application of the boundary conditions (90) to the general solution $z(s)=\sum_{j=1}^{6} a_{j} e^{i k_{j} s}$ leads to the following critical condition:

$$
\begin{aligned}
\chi(\beta):= & 2 \cos \beta+\cos 2 \beta-2\left(\cos \frac{\beta}{2}+\cos \frac{3 \beta}{2}\right) \cosh \frac{\sqrt{3} \beta}{2}+(2-\cos \beta) \cosh \sqrt{3} \beta \\
& -\sqrt{3} \sin \beta \sinh \sqrt{3} \beta-2 \sqrt{3}\left(\sin \frac{\beta}{2}-\sin \frac{3 \beta}{2}\right) \sinh \frac{\sqrt{3} \beta}{2}=0 .
\end{aligned}
$$

The critical loads correspond to pitchfork bifurcations where non-trivial solutions bifurcate from the trivial straight solution. A plot of $\chi$ (Figure 4) shows that in the welded case the pitchfork bifurcations are (doubly) degenerate, as was also found by Wolfe [23]. We stress that the above calculation is only possible for the statics case. If $\omega \neq 0$ then the $x$ and $y$ equations do not decouple and no simple characteristic equation is obtained. 


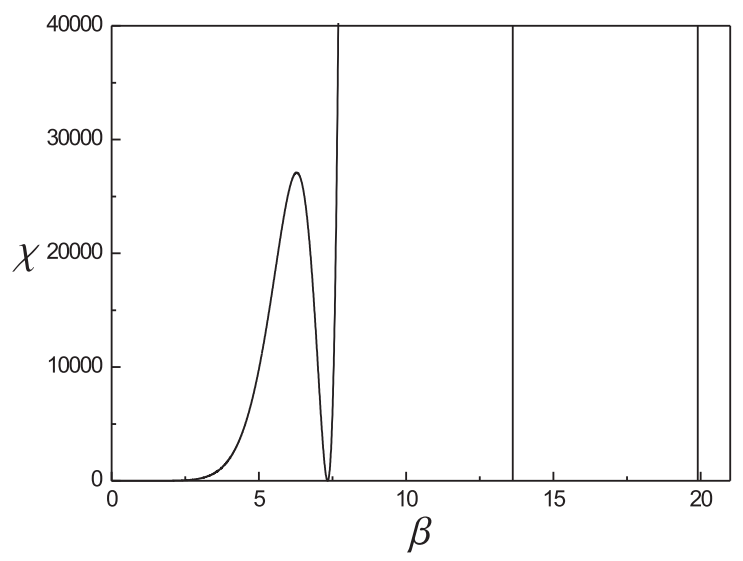

FIG. 4: Degenerate zeroes of the characteristic equation for the welded rod. The first three critical $\beta$ values are $7.332130,13.613561$ and 19.896753 .

B. Eigenvalues for the unperturbed problem $(T=0, \gamma=0, \omega=0, B=0)$

We shall call the case where $T=0, \gamma=0, \omega=0$ and $B=0$ the unperturbed problem. For this problem explicit expressions can be obtained for the eigenvalues of the linearisation about the straight solution. The $x$ and $y$ equations in (87) decouple into two fourth-order beam equations:

$$
\begin{aligned}
& x^{\prime \prime \prime \prime}-\lambda^{2} P x^{\prime \prime}+\frac{\lambda^{2}}{R} x=0, \\
& y^{\prime \prime \prime \prime}-\lambda^{2} P y^{\prime \prime}+\lambda^{2} y=0,
\end{aligned}
$$

subject to boundary conditions (88). Since we anticipate imaginary eigenvalues we set $\lambda=i \mu, x=e^{i k s}, y=e^{i \kappa s}$, and find for the $x$ equation

$$
\begin{aligned}
& k_{1,2}= \pm\left(\frac{1}{2} \mu^{2} P+\frac{1}{2} \sqrt{\mu^{4} P^{2}+4 \mu^{2} / R}\right)^{1 / 2}=: \pm a, \\
& k_{3,4}= \pm i\left(\frac{1}{2} \sqrt{\mu^{4} P^{2}+4 \mu^{2} / R}-\frac{1}{2} \mu^{2} P\right)^{1 / 2}=: \pm i b,
\end{aligned}
$$

while for the $y$ equation

$$
\begin{aligned}
& \kappa_{1,2}= \pm\left(\frac{1}{2} \mu^{2} P+\frac{1}{2} \sqrt{\mu^{4} P^{2}+4 \mu^{2}}\right)^{1 / 2}=: \pm \alpha \\
& \kappa_{3,4}= \pm i\left(\frac{1}{2} \sqrt{\mu^{4} P^{2}+4 \mu^{2}}-\frac{1}{2} \mu^{2} P\right)^{1 / 2}=: \pm i \beta
\end{aligned}
$$


where $a, b, \alpha, \beta$ are non-negative real numbers. The general solutions are

$$
\begin{aligned}
& x(s)=A_{x} \sin a s+B_{x} \cos a s+C_{x} \sinh b s+D_{x} \cosh b s, \\
& y(s)=A_{y} \sin \alpha s+B_{y} \cos \alpha s+C_{y} \sinh \beta s+D_{y} \cosh \beta s .
\end{aligned}
$$

Application of the boundary conditions (88) leads to

$$
\begin{aligned}
& \left(a^{2}-b^{2}\right) \sin a \sinh b=2 a b(1-\cos a \cosh b) \\
& \left(\alpha^{2}-\beta^{2}\right) \sin \alpha \sinh \beta=2 \alpha \beta(1-\cos \alpha \cosh \beta) .
\end{aligned}
$$

In order to obtain the eigenvalues these two transcendental equations can be solved numerically using a Newton-Raphson scheme. Meanwhile, the torsional eigenvalues of the $M_{3}$ equation in (87) are given by

$$
\mu= \pm n \pi \sqrt{\frac{\Gamma}{2 P}} .
$$

These are all the eigenvalues for the unperturbed problem. They will be used as starting values in the numerical procedure described next.

\section{Numerical procedure}

The main idea is to use the known eigenvalues in the unperturbed problem as starting values in a continuation procedure in order to compute the eigenvalues and corresponding eigenfunctions for general values of the parameters $T, \gamma, \omega$ and $B$. For this we use the welltested code AUTO [4] (specifically AUTO2000). AUTO solves boundary-value problems by means of orthogonal collocation. It requires a starting solution and can then trace out solution curves as a parameter of the problem is varied. Bifurcations are detected where branches of solutions intersect. At such points AUTO is able to switch branches and compute curves of bifurcating solutions.

Our procedure takes advantage of the fact that $\lambda$ appears only quadratically in the linearisation (71), (72), (74) and (75) if $\gamma=0$ and $\omega=0$. To explain the method consider the typical $O(\delta)$ equation

$$
z^{\prime \prime \prime \prime}-\lambda^{2} f(s) z^{\prime \prime}+\lambda^{2} g(s) z=0
$$

where $f$ and $g$ are functions of the $O(1)$ solution. Writing $z=x+i y, \lambda=\lambda_{r}+i \lambda_{i}$, we can 
decompose the $z$ equation into

$$
\begin{aligned}
& x^{\prime \prime \prime \prime}-\left(\lambda_{r}^{2}-\lambda_{i}^{2}\right) f(s) x^{\prime \prime}+2 \lambda_{r} \lambda_{i} f(s) y^{\prime \prime}+\left(\lambda_{r}^{2}-\lambda_{i}^{2}\right) g(s) x-2 \lambda_{r} \lambda_{i} g(s) y=0, \\
& y^{\prime \prime \prime \prime \prime}-\left(\lambda_{r}^{2}-\lambda_{i}^{2}\right) f(s) y^{\prime \prime}-2 \lambda_{r} \lambda_{i} f(s) x^{\prime \prime}+\left(\lambda_{r}^{2}-\lambda_{i}^{2}\right) g(s) y+2 \lambda_{r} \lambda_{i} g(s) x=0 .
\end{aligned}
$$

The important thing to note here is that these equations decouple into two identical equations if the eigenvalue is either imaginary $\left(\lambda_{r}=0\right)$ or real $\left(\lambda_{i}=0\right)$.

This suggests the following sequence of steps, involving boundary-value problems of increasing dimension, to compute eigenvalues of statical or uniformly whirling solutions.

1. Consider the unperturbed problem of Section VIB and, noting that all eigenvalues are purely imaginary, solve the 30-dimensional system of $18 O(1)$ equations and one 12dimensional system for the imaginary part of the $O(\delta)$ equations (cf. the $y$ equation in (94)). Set $\lambda_{r}=0$ and use $\lambda_{i}$ as the continuation parameter in AUTO in order to compute the eigenvalues (instead of solving the transcendental equations in Section VIB). These eigenvalues will show up as branching points (BP), or pitchfork bifurcations, as eigenvalues by definition are those values for which non-zero BVP solutions exist. By symmetry it is only necessary to consider $\lambda_{i}>0$.

2. Keeping the same 30-dimensional system, switch branches at a BP to compute ('grow') the corresponding (imaginary) eigenfunction. Since the equations are linear the value of $\lambda_{i}$ will not change in this run. For later use we monitor the non-zero solution by means of some measure $\|.\|_{i}$ (not necesarily a proper norm) on the space $\left\{\hat{\boldsymbol{x}}_{i}^{t}, \hat{\boldsymbol{\alpha}}_{i}^{t}, \hat{\boldsymbol{F}}_{i}^{t}, \hat{\boldsymbol{M}}_{i}^{t}\right\}$ of imaginary linearised variables.

3. Now consider the full system of 42 equations $(18 O(1)$ equations and two sets of 12 dimensional $O(\delta)$ equations (cf. (94)). Fix the measure $\|\cdot\|_{i}$ on the imaginary part and release $\lambda_{r}$ instead in order to compute the real eigenfunction (since the imaginary part of the solution is fixed there is only one branch of solutions through the starting point and there is nowhere else to go for the continuation but to 'grow' the real eigenfunction). Again we monitor this function by means of a suitable measure $\|.\|_{r}$. In this run neither $\lambda_{r}$ nor $\lambda_{i}$ will change.

This approach works because the solution obtained in step 2 also solves the full 42 dimensional system when the extra 12 variables $\left(\hat{\boldsymbol{x}}_{r}^{t}, \hat{\boldsymbol{\alpha}}_{r}^{t}, \hat{\boldsymbol{F}}_{r}^{t}, \hat{\boldsymbol{M}}_{r}^{t}\right)$ are set to zero. This is a consequence of the fact that the real and imaginary parts of the $O(\delta)$ equations 
decouple if $\lambda_{r}=0$, as a result of the quadratic dependence of the eigenvalue problem on $\lambda$ (cf. (94)).

Steps 2 and 3 can be performed for as many of the BPs computed in step 1 as required and will give the corrsponding eigenvalues and eigenfunctions. Once these have been obtained both measures $\|.\|_{r}$ and $\|.\|_{i}$ can be fixed and an extra system parameter such as $B$ or $\omega$ released in order to trace the eigenvalues (and hence monitor stability changes) as system parameters are varied. (Note that fixing $\|.\|_{r}$ and $\|.\|_{i}$ makes sense as eigenfunctions are only defined up to a multiplicative factor.)

The above 3-step procedure is not limited to linearisations about the trivial straight solution. It can be applied to any starting solution that has no eigenvalue with both $\lambda_{r}$ and $\lambda_{i}$ non-zero, as these would not be picked up in step 1. (It is of course no problem if eigenvalues become fully complex (e.g., in a Hopf bifurcation) in the course of further continuations.) For instance, we find that at the first critical $B$, given by (91), the lowest conjugate pair of eigenvalues $\pm \lambda_{i}$ goes to zero and becomes a real pair of eigenvalues, signalling a stability change of the straight rod. The (first-mode) solution bifurcating at this point is stable with all eigenvalues being imaginary and the above procedure can be applied to find the eigenvalues.

We end this section with a few comments:

(i) There are infinitely many eigenvalues and the above procedure only finds the lowest order ones. This is of course a limitation of any numerical scheme. We find that eigenvalues vary slowly with system parameters, suggesting that stability is governed by the lowest-order eigenvalues. We typically consider 5 or 6 eigenvalues.

(ii) Note that in steps 1 and 2 above we could not have taken the full 42-dimensional system of equations as that would have made the branching points (pitchfork bifurcations) degenerate and AUTO would not detect a BP. This is because if $\lambda_{r}=0$ (or $\lambda_{i}=0$ ) the two sets of 12-dimensional linearised equations are identical (cf. (94)). 
TABLE I: Dimensional and dimensionless parameters for the SET.

\begin{tabular}{|c|c||c|c|}
\hline$L$ & $100 \mathrm{~m}$ & $P$ & 0.001 \\
\hline$A$ & $2.879 \times 10^{-11} \mathrm{~m}^{2}$ & $R$ & 0.5526 \\
\hline$E$ & $1.32 \times 10^{11} \mathrm{~N} / \mathrm{m}^{2}$ & $\Gamma$ & 0.76923 \\
\hline$E I_{1}$ & $38 \mathrm{Nm}^{2}$ & $f$ & 500.5639 \\
\hline$E I_{2}$ & $21 \mathrm{Nm}^{2}$ & & \\
\hline
\end{tabular}

\section{NUMERICAL RESULTS}

\section{A. The statics case, $\omega=0$}

Figures 5 and 6 show the bifurcation diagram obtained when the magnetic field parameter $B$ is varied, using different measures along the vertical axes. The dimensionless parameters taken are those of the SET and are listed in Table I, along with the dimensional parameters. We observe that at critical values of $B$ pairs of non-trivial solution branches bifurcate, thus confirming the degenerate nature of the bifurcation found analytically in Section VIA. The first three bifurcations occur at $B=0.585(B P 1), 3.747(B P 2)$ and $11.698(B P 3)$, in agreement with Figure 4 when the numerical factor $f$ is taken into account. In addition to these primary branches, there are branches, labelled b12, b34 and b56, connecting these pairs. One end of these connecting branches reaches down to the horizontal axis, while the other end comes out of a secondary pitchfork bifurcation along the first branch of the pair of primary branches. They in fact come in symmetric pairs as is brought out by the measure plotted in Figure 6. The shapes of the rod along the connecting branches form a smooth transition between the shapes on the connected branches; see Figure 7 where projections onto the $\left\{\boldsymbol{e}_{1}-\boldsymbol{e}_{3}\right\}$ and $\left\{\boldsymbol{e}_{2}-\boldsymbol{e}_{3}\right\}$ planes of bifurcating solutions along branches b1, b2 and b12 are shown at constant measure ${ }_{1}=0.04$.

Solutions bifurcating at larger $B$ values have successively more coils. Figure 8 shows the three-dimensional shape of a solution on the fifth bifurcating branch.

Figure 9 shows the evolution of the imaginary and real parts of the first five pairs of eigenvalues along the trivial solution, from $B=0$ to $B>B P 2$ (i.e., beyond the second pitchfork). As the branches are born in pairs at the degenerate pitchfork, two pairs of pure 


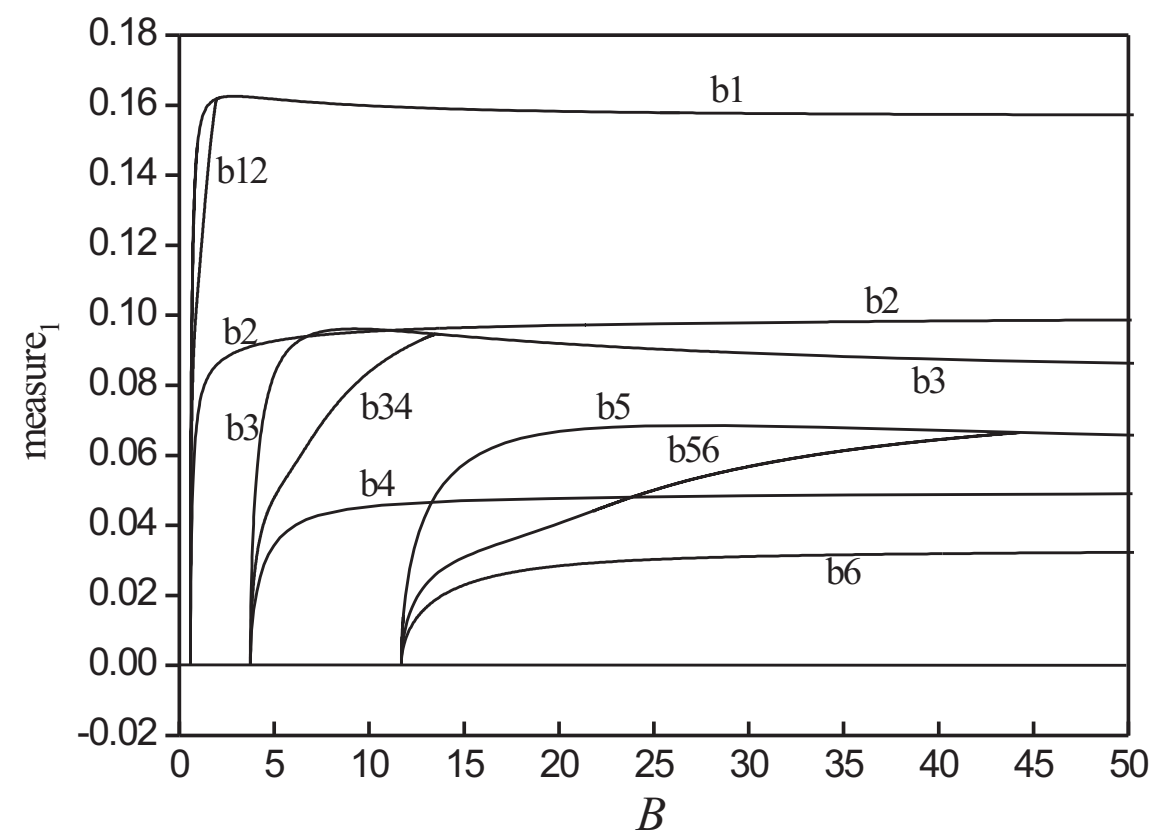

FIG. 5: Bifurcation diagram for the statical case $(\omega=0)$. System parameters are those of Table 1 . measure $_{1}=\int_{0}^{1}\left|x^{0}(s)\right| \mathrm{d} s$.

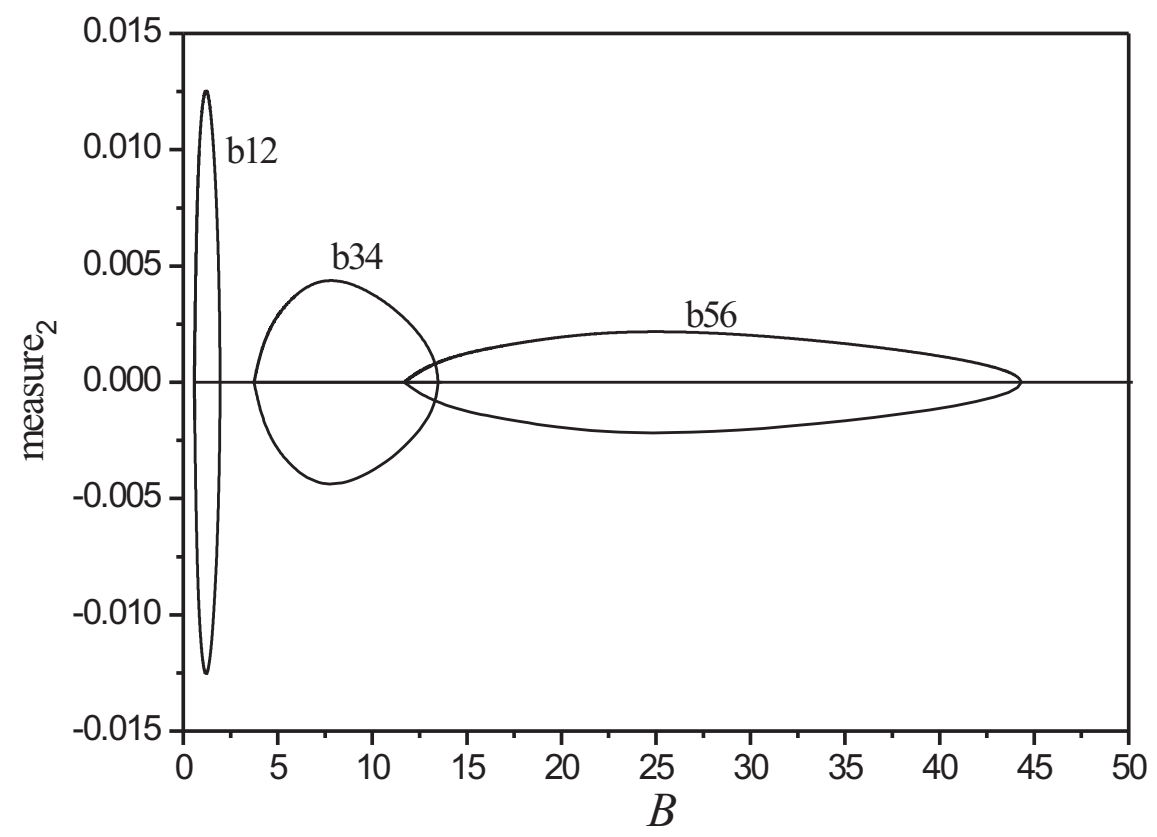

FIG. 6: Bifurcation diagram for the statical case $(\omega=0)$ using a different solution measure. measure $_{2}=\int_{0}^{1} x^{0}(s) y^{0}(s) \mathrm{d} s$. The primary branches have measure $2=0$. 

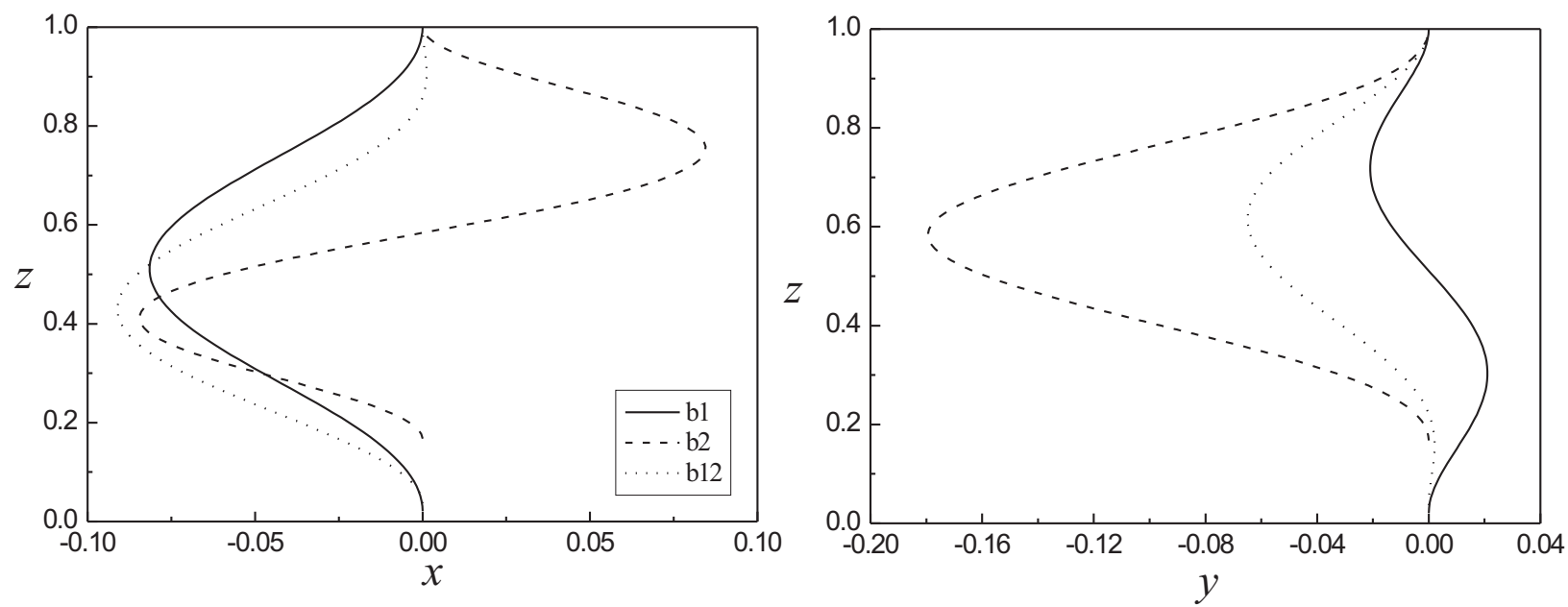

FIG. 7: Projection of the modes corresponding to branches b1, b2 and transition branch b12 onto the $\left\{\boldsymbol{e}_{1}-\boldsymbol{e}_{3}\right\}$ and $\left\{\boldsymbol{e}_{2}-\boldsymbol{e}_{3}\right\}$ planes at constant measure ${ }_{1}=0.04$. Values of $B$ are: 0.595 (b1), 0.605 (b12) and $0.7(\mathrm{~b} 2)$.

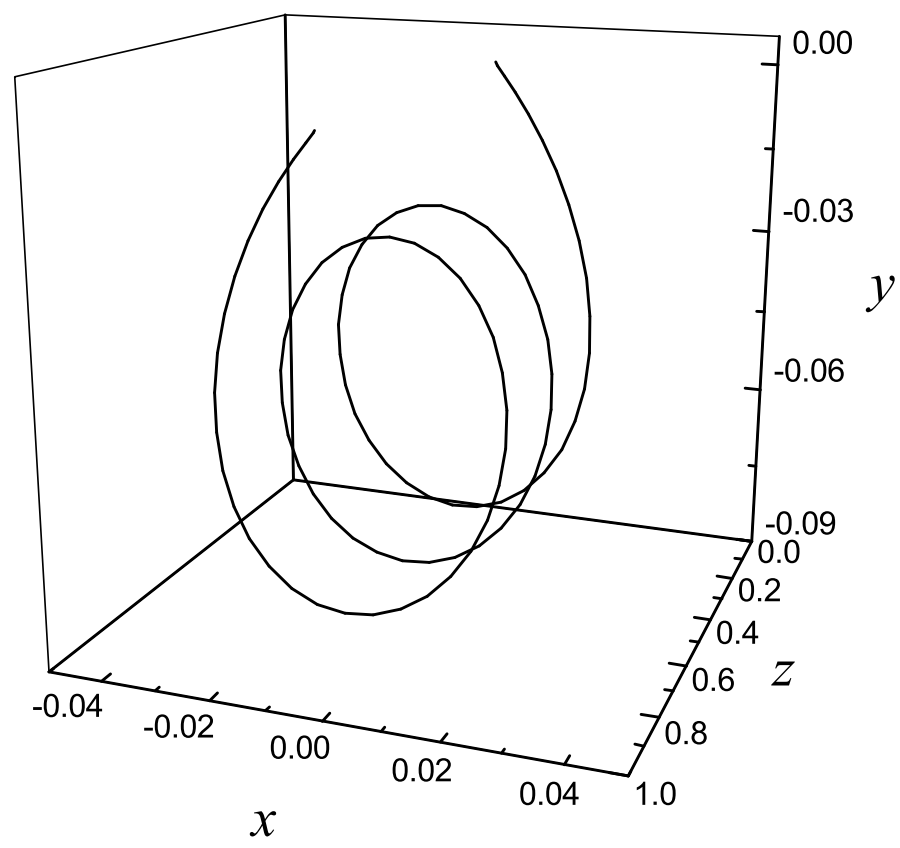

FIG. 8: 3D view of a solution along branch b5.

imaginary eigenvalues collide at zero and become pure real pairs at $B=0.585(B P 1)$. One of the eigenvalues of each real pair is positive, meaning that the trivial solution becomes unstable at $B=B P 1$, as one would expect. A similar further loss of stability occurs at 
(a)

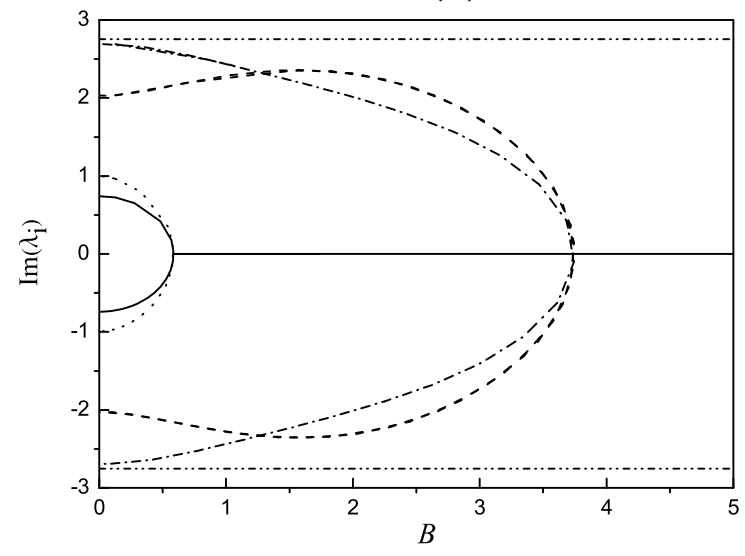

(b)

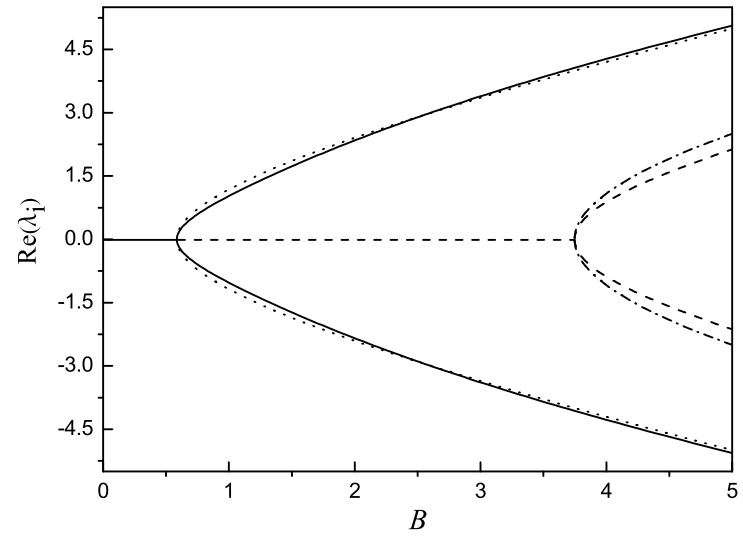

FIG. 9: Evolution of imaginary (a) and real (b) part of the first five pairs of eigenvalues along the trivial solution as $B$ is increased from 0 to $>B P 2$.

$B=B P 2$.

Figure 10 shows the evolution of the first five pairs of purely imaginary eigenvalues when switching from the stable trivial branch to branch b1, which is initially found to be stable. The figure reveals that branch b1 loses stability at the secondary pitchfork bifurcation at $B=1.942$, where b12 connects. At this point the first pair of eigenvalues becomes real, as illustrated in Figure 11. This figure also shows that connecting branch b12 is unstable as it too has a pair of real eigenvalues.

Considering the problem of the ET from a design point of view, it is interesting to quantify the value of the geomagnetic field at which the straight tether would buckle. In the case at hand, this value is $B=0.587$ (see Figure 5), which in dimensional parameters yields $I B_{0}=2.104 \times 10^{-5} \frac{\mathrm{N}}{\mathrm{m}}$. Noting that the maximum value of the geomagnetic field is $B_{g}=7 \times 10^{-5} T[11$, and assuming that the maximum current that will flow along the tether would be $I=1 A[11]$, the maximum expected value for the constant $I B_{g}=7 \times 10^{-5} \frac{\mathrm{N}}{\mathrm{m}}$. This means that the ET would be below the critical value at any time. Note, though, that $B$ goes as the cube of $L$ and for longer tethers, which are common in radially stabilised ETs, the critical value may be exceeded resulting in buckling into a coiled shape (cf. Figure 8), as has been reported in some tether flights such as the PGM and TSS-1R missions [2, 11]. 


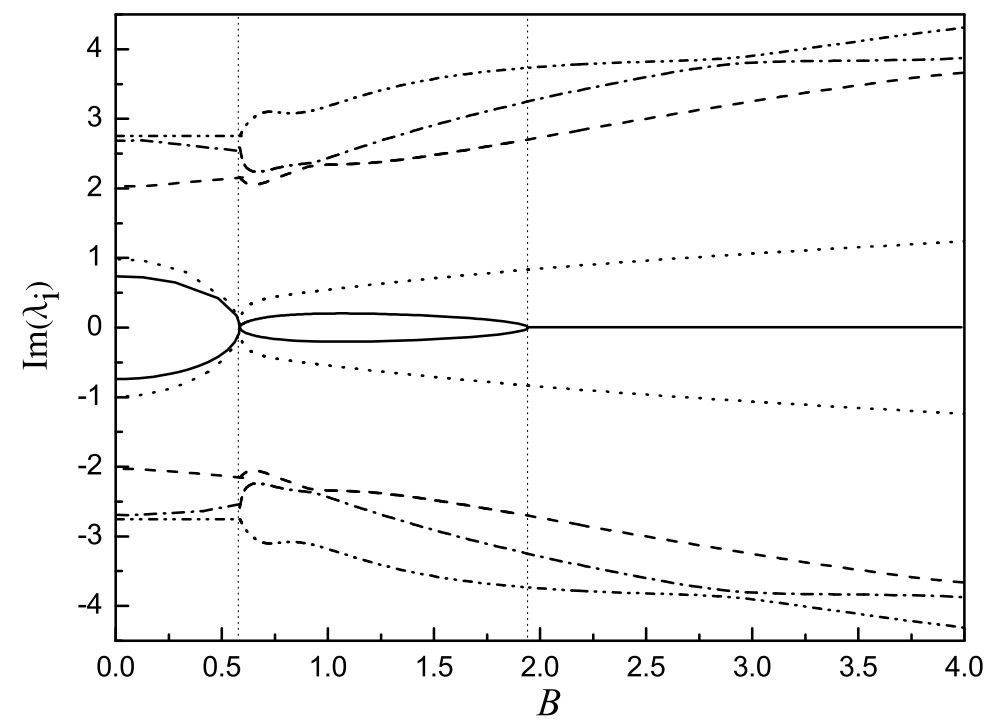

FIG. 10: Imaginary part evolution of the first five pairs of eigenvalues along the trivial solution from $B=0$ to $B=B P 1$, then following branch b1 beyond the secondary pitchfork.

\section{B. Whirling solutions (relative equilibria), $\omega \neq 0$}

We now introduce angular velocity $\omega$ to the ET and seek relative equilibria, that is, solutions that appear static when viewed from the rotating frame $\left\{\boldsymbol{e}_{1}, \boldsymbol{e}_{2}, \boldsymbol{e}_{3}\right\}$. Figures 12 , 13 and 14 show the effect of increasing angular velocity on the bifurcation diagram of Figure 5 . The degenerate pitchfork bifurcations get resolved and the connecting branches move up along the principal branches. For $\omega=0.25$ (Figure 12) all branches, b1, b2, ..., bifurcate, as in the statics case of Figure 5, and the trivial solution is stable up to the first primary pitchfork. However, at $\omega=0.75$ (Figure 13), b1 has merged with its symmetric partner at $B<0$ and lifted off the horizontal axis, leaving no stable straight solutions. b2 similarly lifts off near $\omega=1.25$. Figure 14 shows the bifurcation diagram at $\omega=2$. An extra branch of connecting solutions (b22) has appeared, but no further branches have become non-bifurcating.

Figure 15 shows the bifurcation diagram at $\omega=2$ for the case of an isotropic rod $(R=1)$. Here the bifurcations become degenerate again but the connecting branches do not move down. In fact, they turn into vertical branches. We conclude that neither anisotropy $(R)$ nor whirl $(\omega)$ alone resolves the pitchfork degeneracy; both are required.

To investigate the stability of non-trivial solutions we follow the eigenvalues computed 


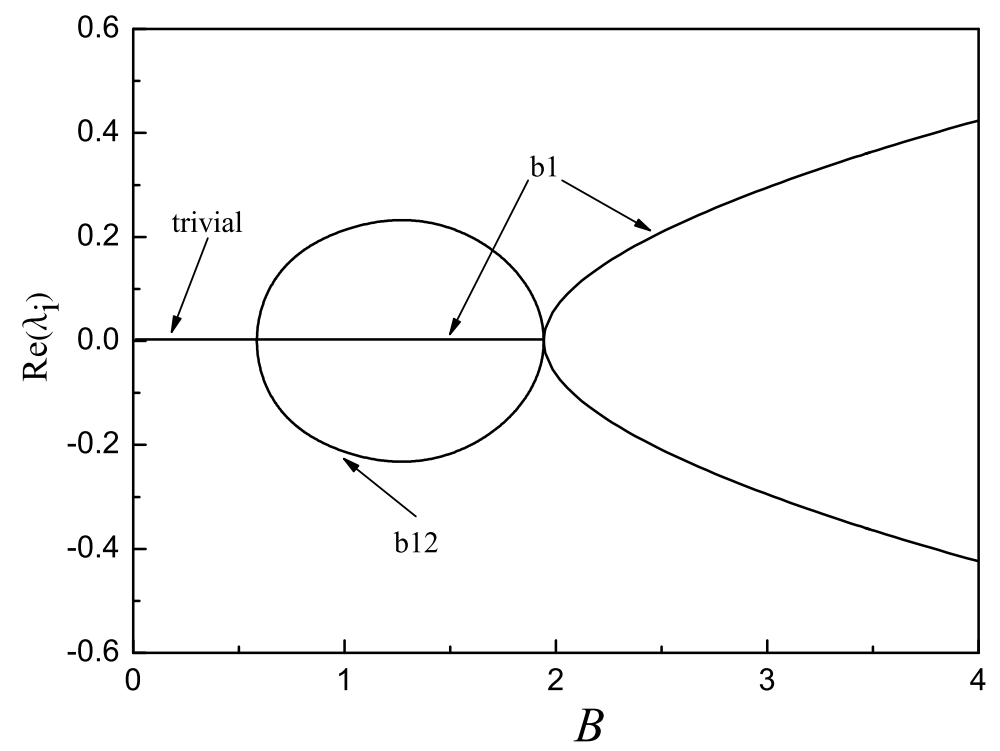

FIG. 11: Real part evolution of the first pair of eigenvalues along the trivial solution from $B=0$ to $B=B P 1$, then following branch b1 beyond the secondary pitchfork as well as connecting branch b12.

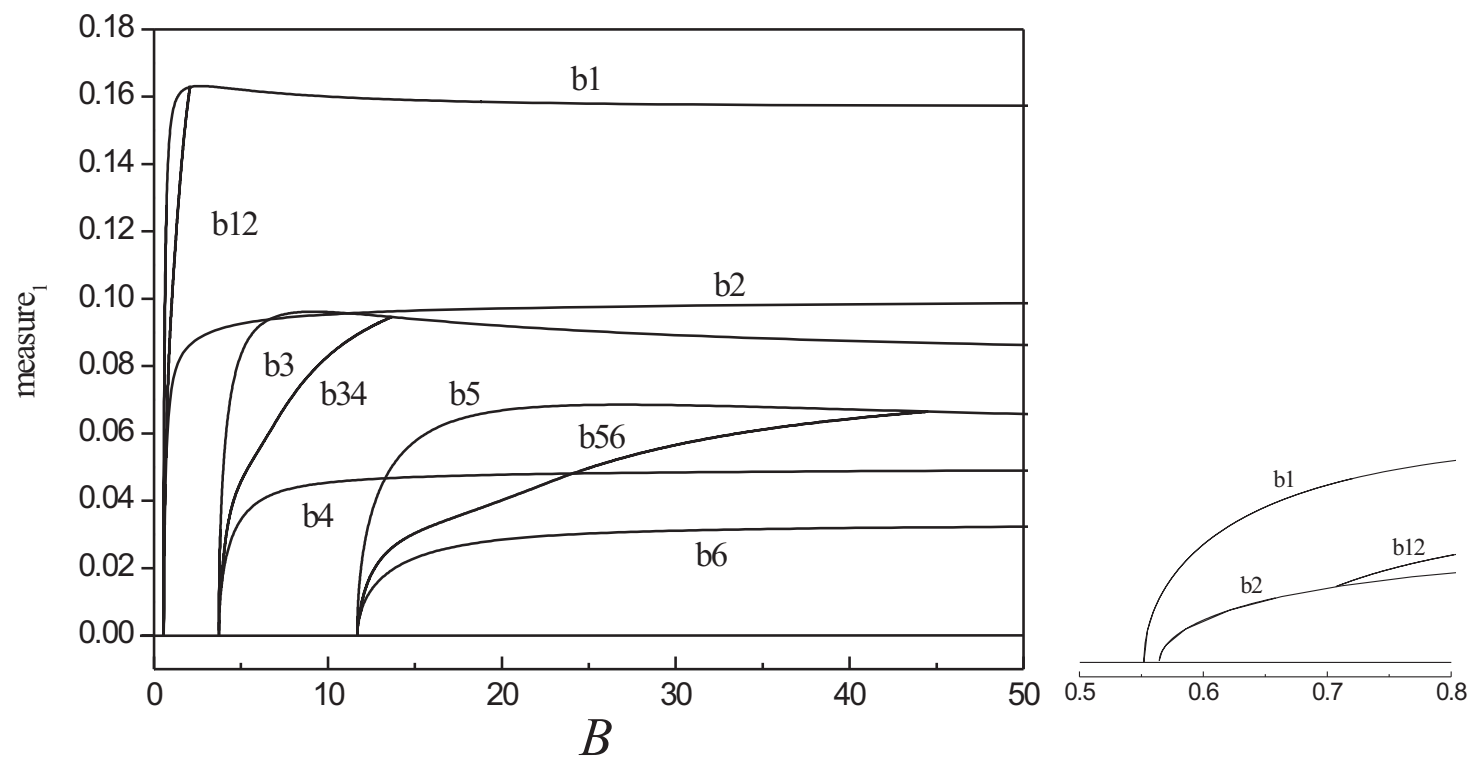

FIG. 12: Bifurcation diagram for a whirling anisotropic rod at $\omega=0.25$. Branches b1 and b2 still bifurcate (at distinct values of $B$ ) and trivial solutions before the first pitchfork are stable. 

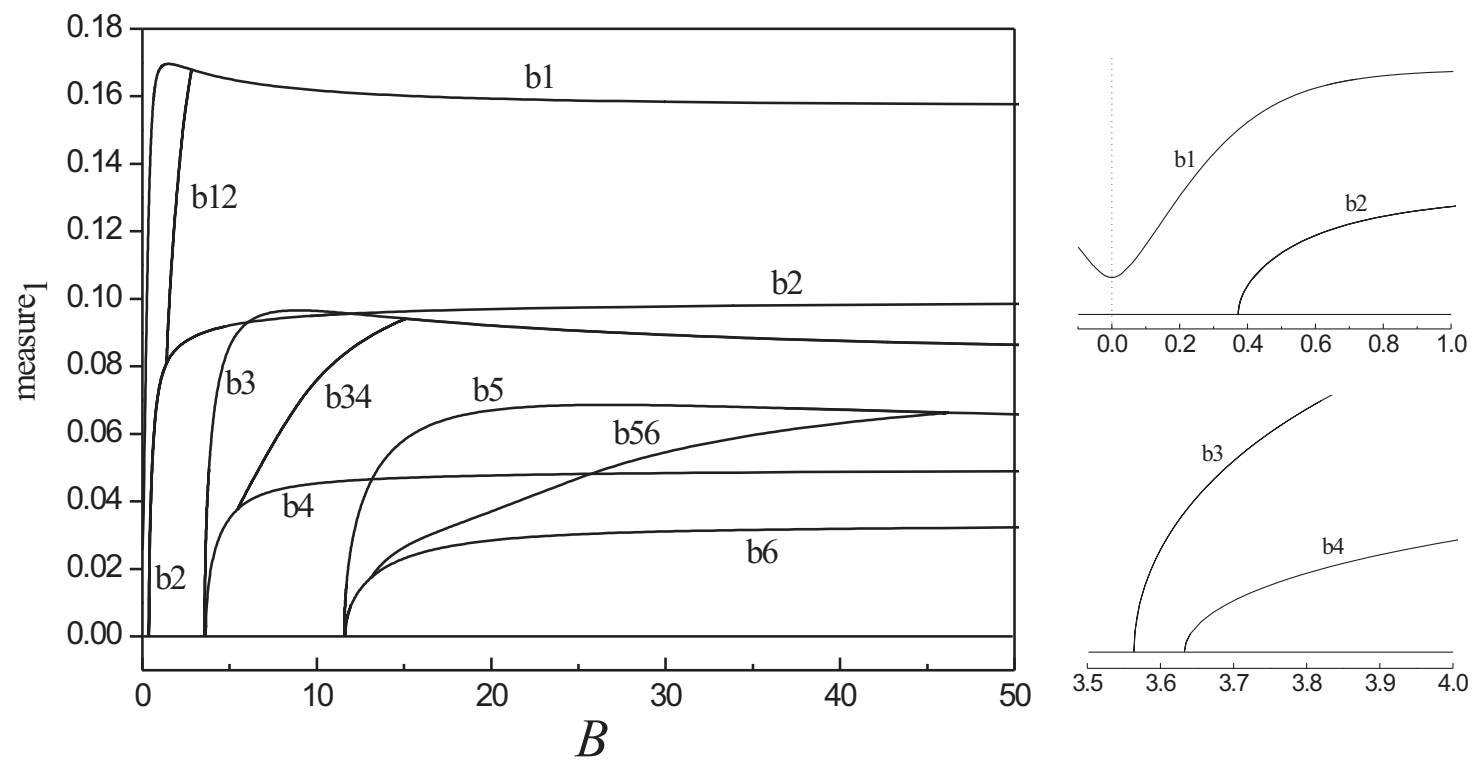

FIG. 13: Bifurcation diagram for a whirling anisotropic rod at $\omega=0.75$. Branch b1 no longer bifurcates and all trivial solutions are unstable.

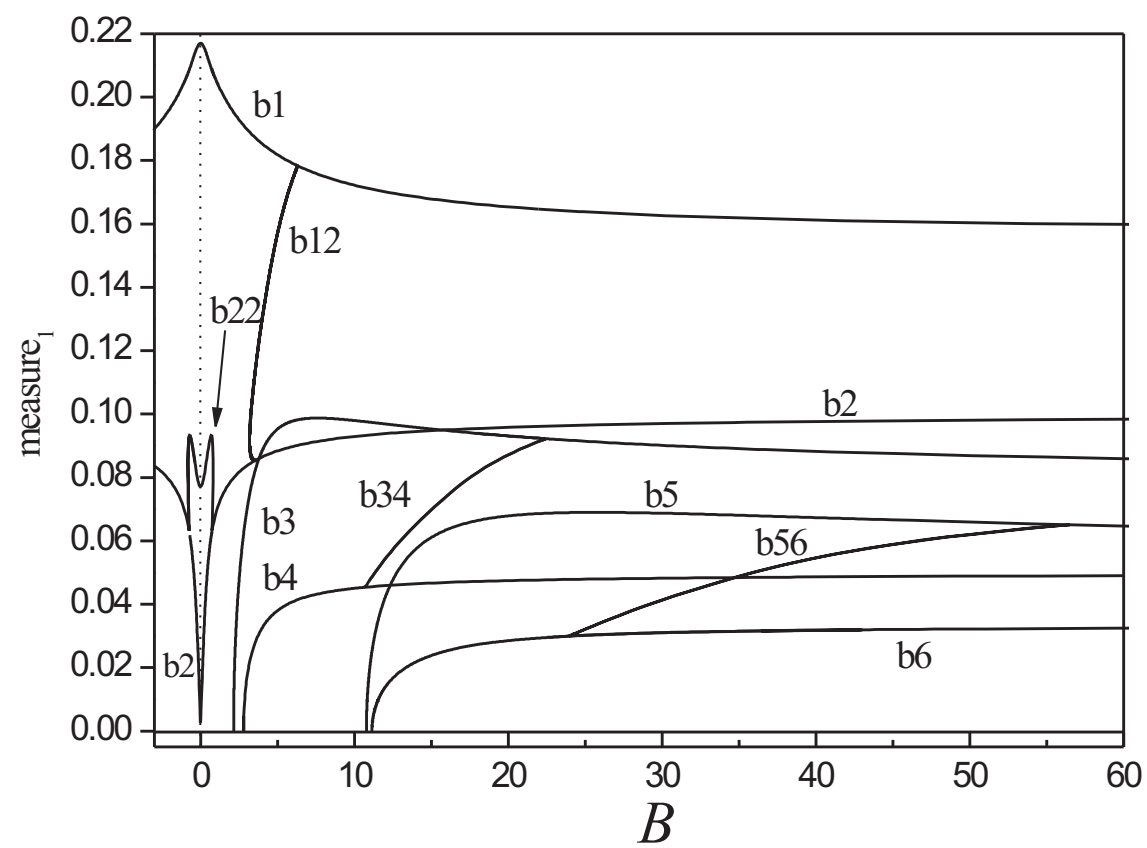

FIG. 14: Bifurcation diagram for a whirling anisotropic rod at $\omega=2$. Branches b1 and b2 no longer bifurcate. An extra (unstable) branch b22 has appeared. 


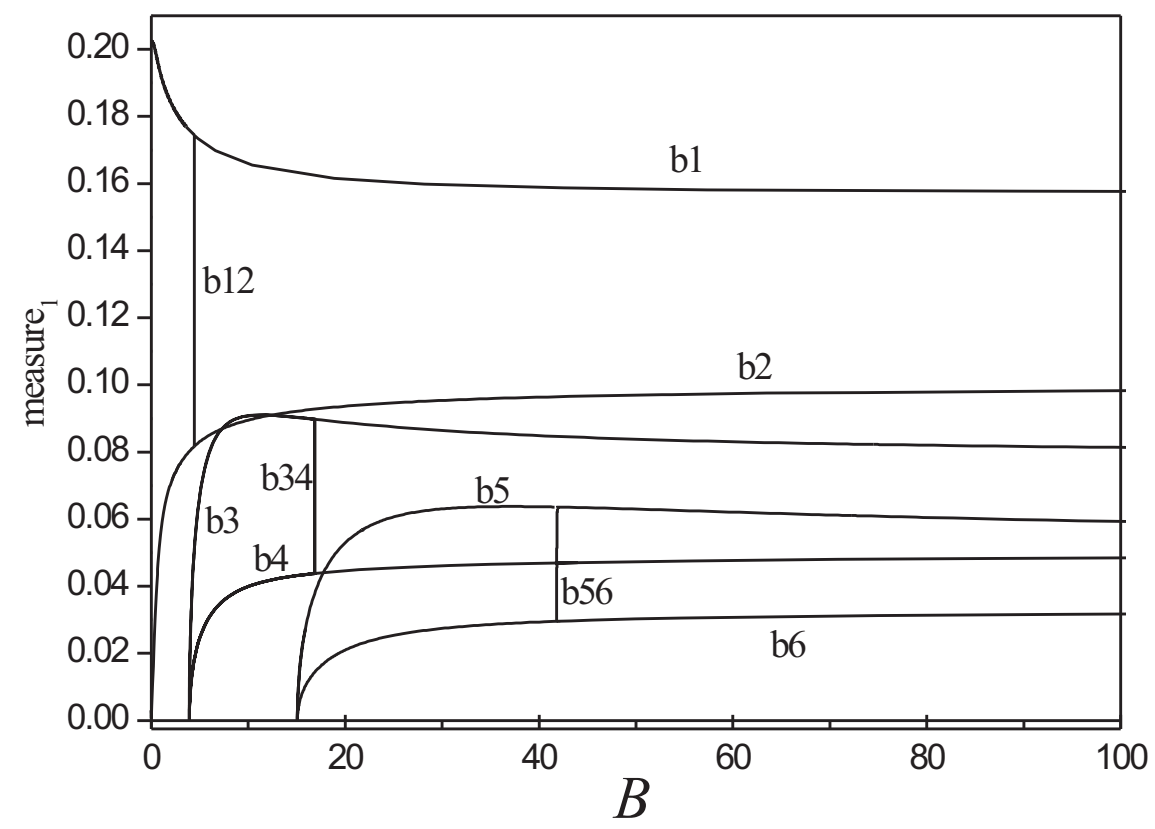

FIG. 15: Bifurcation diagram for a whirling isotropic $\operatorname{rod}(\omega=2, R=1)$.

(a)

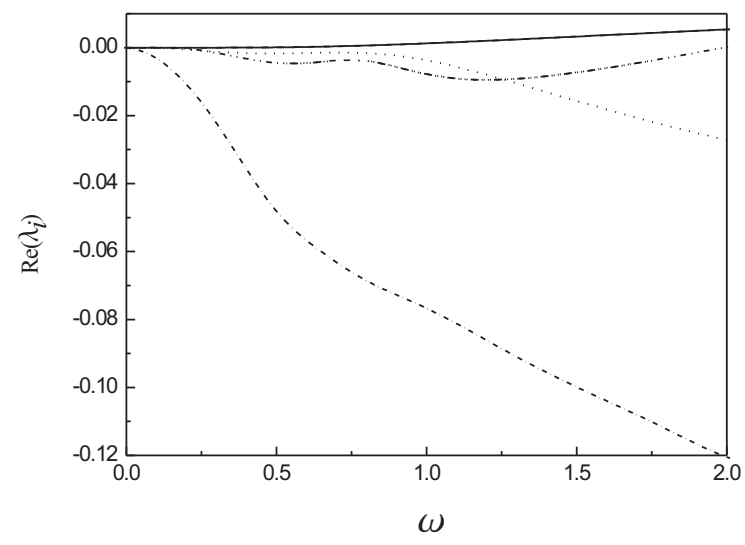

(b)

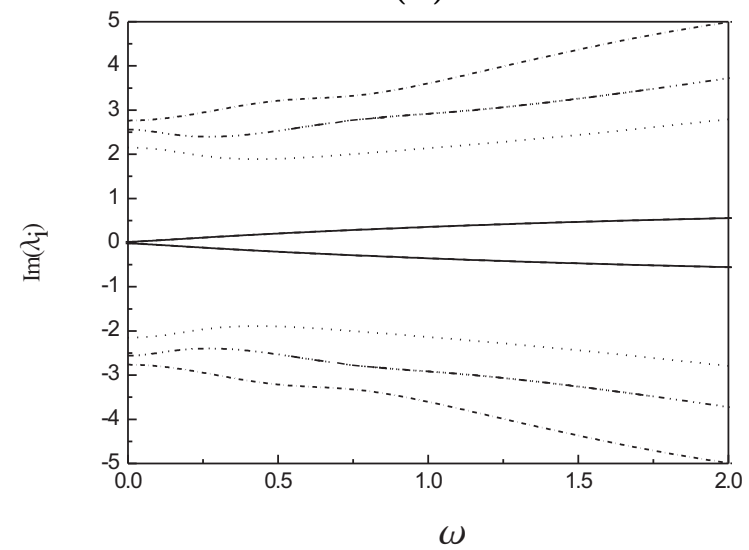

FIG. 16: Evolution with $\omega$ of real (a) and imaginary (b) parts of the first five pairs of eigenvalues from branch b1 of Figure $5(\omega=0)$ with $B=0.5869$.

earlier as $\omega$ is increased from 0. Figure 16 shows the evolution of the real and imaginary parts of the first five conjugate pairs of eigenvalues when continuing in $\omega$ from a static solution on the first branch (b1) of Figure 5 at fixed $B=0.5869$. Note that the first and second eigenvalues are almost coincident and can hardly be distinguished in the figure. Both eigenvalues acquire positive real parts; hence the static coiled tether becomes unstable as 

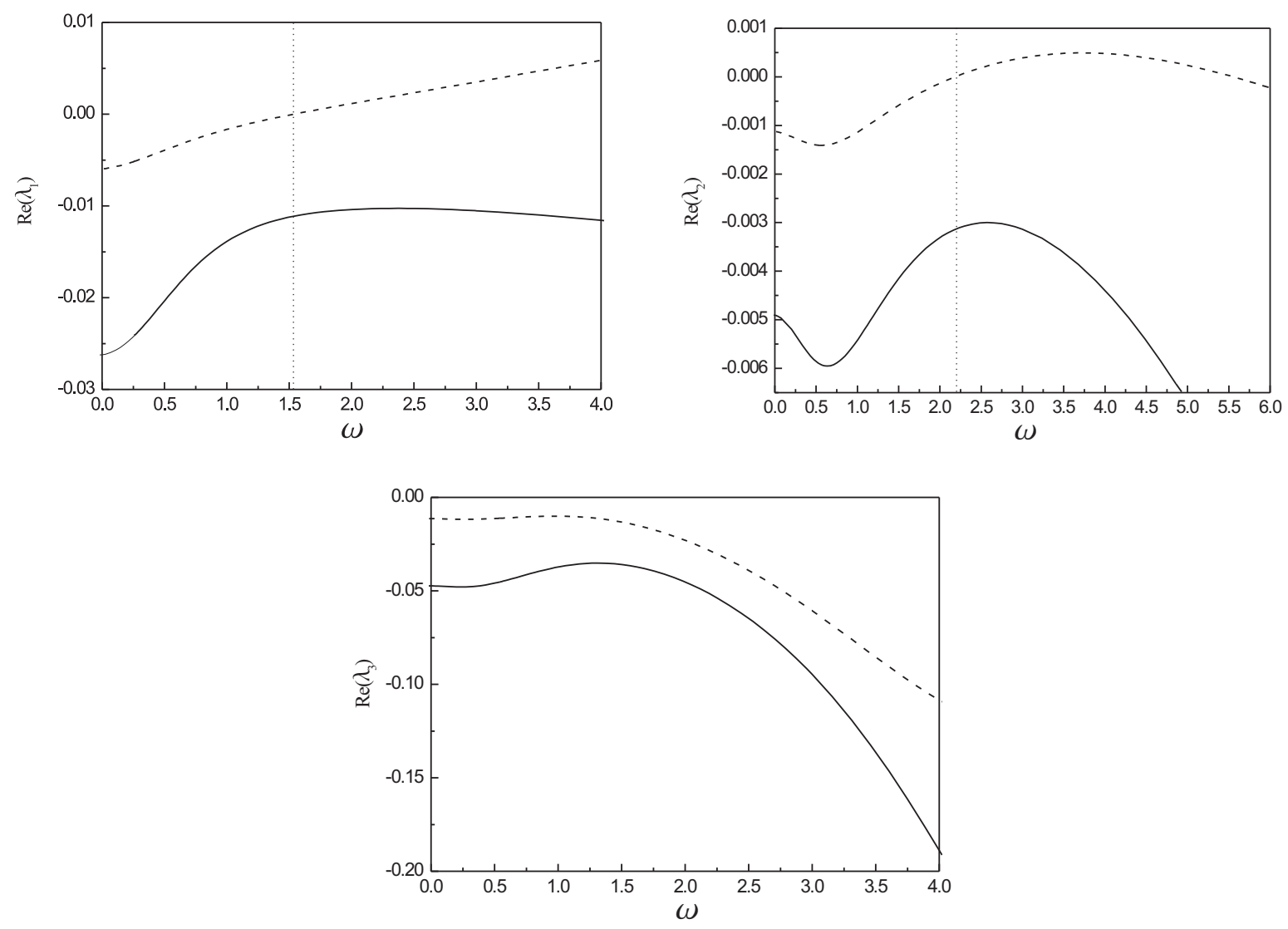

FIG. 17: Evolution with respect to $\omega$ of the real part of the first three pairs of eigenvalues for a solution along b1 at $\gamma=0.04375$ (solid) and $\gamma=0.01$ (dashed). $(B=1.2$.)

soon as it is being rotated about $\boldsymbol{k}$. The other eigenvalues move to the negative real halfplane as $\omega$ is increased. The angular velocity is increased to $\omega=2$, which is beyond the critical velocity in the $B=0$-case where the tether buckles at the first bending natural frequency of the beam, $\omega=\sqrt{R}[19]$.

Next we consider the combined effect of both damping and spin on the stability of the rod. Figure 17 and 18 show the evolution of the first three eigenvalues as $\omega$ is increased for a solution along the first bifurcating branch b1 at constant $B=1.2$ and two different values of the damping coefficient. The value $\gamma=0.04375$ was estimated by Valverde et al. [19] to be the damping value for a real electrodynamic tether under working conditions. The figures show that the solution remains stable at least up to $\omega=4$ (higher-order eigenvalues do not seem to alter this picture). However, at the lower value of $\gamma=0.01$ we observe a Hopf bifurcation at $\omega \approx 1.5$ where the conjugate pair of eigenvalues $\left(\lambda_{1}, \bar{\lambda}_{1}\right)$ acquires a positive 

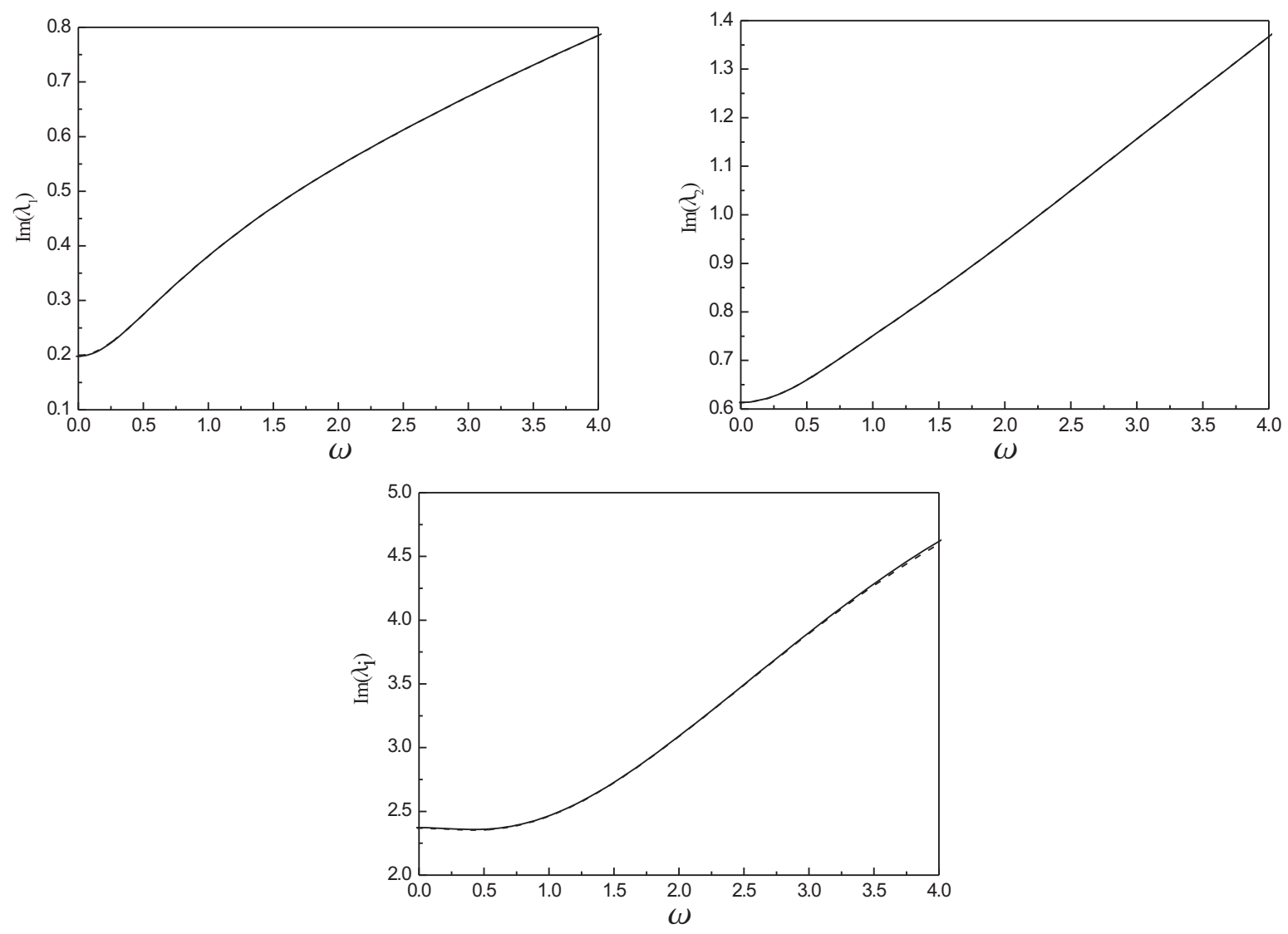

FIG. 18: Evolution with respect to $\omega$ of the imaginary part of the first three pairs of eigenvalues for a solution along b1 at $\gamma=0.04375$ (solid) and $\gamma=0.01$ (dashed). The curves are independent of $\gamma \cdot(B=1.2$.

real part (at $\omega \approx 2.2$ the second pair of eigenvalues $\left(\lambda_{2}, \bar{\lambda}_{2}\right)$ moves into the positive real half plane, returning to the negative half plane at $\omega \approx 6$ ). Also note that damping only affects the real parts of the eigenvalues, not the imaginary parts (Figure 18).

Figure 19] shows curves of these Hopf bifurcations, both of $\lambda_{1}$ and $\lambda_{2}$, in the $B-\omega$ parameter plane for various values of $\gamma$, as well as the curve of secondary pitchfork bifurcations. The latter curve is independent of $\gamma$. This makes sense as our (quasi-) statics problem, and hence the bifurcation diagrams in Figures [12, 13] and 14, do not depend on $\gamma$, and therefore the secondary pitchfork bifurcations, where a real eigenvalue goes through zero, must also be independent of $\gamma$. Interestingly, the Hopf bifurcation curves of $\lambda_{1}$ are closed, with instability inside the curves, while the Hopf bifurcation curves of $\lambda_{2}$ are open. The closed curves shrink to zero at $\gamma=0.023$, leaving only instability due to Hopf bifurcations of $\lambda_{2}$ for larger values 


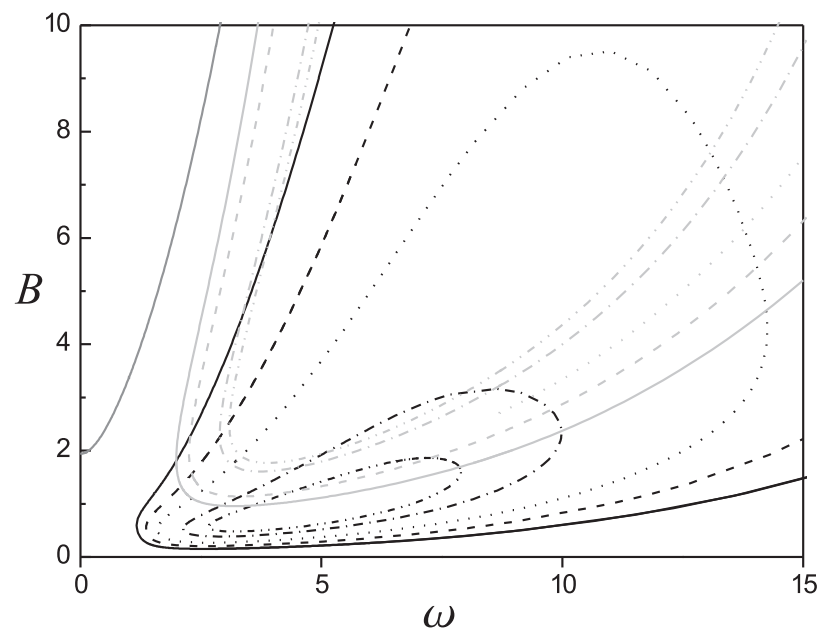

FIG. 19: Curves of secondary pitchfork bifurcations (dark grey), Hopf bifurcations of $\lambda_{1}$ (black) and Hopf bifurcations of $\lambda_{2}$ (light grey) at $\gamma=0.01,0.013,0.016,0.02$ and 0.022 (from outside to inside). The stable region is below the dark grey curve and around the black and light grey knees.

of $\gamma$. In the limit $\gamma \rightarrow 0$ the Hopf curves approach the coordinate axes $\omega=0, B=0$. The region of stable b1 solutions in the diagram is the connected L-shaped region between the secondary pitchfork and Hopf curves.

\section{CONCLUSION}

We have shown that whirling current-carrying rods bifurcate under increasing magnetic field (or current). For the welded boundary conditions considered here this bifurcation is doubly degenerate (even for a transversely isotropic rod). This is because the equations are invariant under rotation about the axis ( $\boldsymbol{e}_{3}$ axis) of the supports. This symmetry property complicated Wolfe's analysis, both for the whirling string [22] and the statical rod [23]. In both models account had to be taken of the variational nature of the problem to prove existence of non-trivial bifurcating states. We find that for the rod, eigenvalues become simple when rotating states are considered, provided the rod is anisotropic. Neither spin nor anisotropy alone resolves the degeneracy.

Wolfe's analysis [21] for non-whirling strings suggests that the first bifurcating branch is stable and that all other branches are unstable. We find the same to be true for a welded rod, initially, but a secondary pitchfork bifurcation occurs where the post-buckling solution 
(along b1) loses stability. Unfortunately, our method allows us only to study stationary or quasi-stationary (whirling) solutions, so it is not clear what type of solutions occur when the (quasi-) stationary solutions lose stability in these secondary bifurcations. To investigate this one would have to do simulations based on direct discretisation of the PDEs (19) and (20).

Associated with the secondary pitchfork bifurcations are connecting orbits (b12, b34, etc.) which interfere with the primary pitchfork bifurcations in the limit $\omega \rightarrow 0$. Another interesting bifurcation scenario concerns the merging of symmetrically related branches (at $B=0)$ and the subsequent 'lifting off' of these branches, thereby becoming non-bifurcating. In the case of b1 (Figure 13) this leads to the disappearance of stable trivial (straight) solutions.

We have performed the, as far as we are aware, first study of the magnetically induced post-buckling behaviour of elastic rods. Our results may be of interest to the design of static as well as spinning electrodynamic tethers, which often operate in the post-buckling regime. As we pointed out in Section 7.1, the critical values of the magnetic field (or current) that we find may be close to operating values for certain tether types. Furthermore, axial spin tends to destabilise the post-buckling state of the tether through Hopf bifurcations, although typical viscoelastic material damping levels go some way towards avoiding Hopf instabilities. On the other hand, spin has a stabilising effect in that it tends to push the secondary pitchfork bifurcation to higher values of the magnetic field. We have mapped out stable regions in the $B-\omega$ parameter plane (Figure 19).

We finally like to speculate on another possible application of this work. There is great current interest in conducting nanowires. These can either be silicon-based wires, carbon nanotubes or metal-coated biological fibres such as proteins, DNA molecules and microtubules [16]. In addition there is the ongoing discussion whether or not DNA molecules are electrical conductors [12]. All these nanometer-scale structures are believed to have great potential as building blocks for future electronic devices. The interaction of these wires with magnetic fields could conceivably be exploited to obtain certain desirable properties.

[1] Antman, S.S., 1995. Nonlinear Problems of Elasticity. Springer-Verlag, Berlin.

[2] Beletsky, V.V. and Levin, E.M., 1993. Dynamics of Space Tether Systems. Advances in the 
Astronautical Sciences Volume 83, American Astronautical Society.

[3] Coleman, B.D., Dill, E.H., Lembo, M., Lu, Z. and Tobias, I., 1993. "On the dynamics of rods in the theory of Kirchhoff and Clebsch". Arch. Rat. Mech. Anal. 121, pp. 339-359.

[4] Doedel, E.J., Champneys, A.R., Fairgrieve, T.R., Kuznetsov, Yu.A., Sandstede, B. and Wang, X.J., 1997. AUTO97: Continuation and bifurcation software for ordinary differential equations (available by anonymous ftp from ftp.cs.concordia.ca/pub/doedel/auto).

[5] Fraser, W.B. and Stump, D.M., 1998. "Yarn twist in the ring-spinning balloon". Proc. R. Soc. Lond. A 454, pp. 707-723.

[6] Goriely, A. and Tabor, M., 1997. "Nonlinear dynamics of filaments I. Dynamical instabilities". Physica D 105, pp. 20-44.

[7] Healey, T.J., 1990. "Large rotating states of a conducting elastic wire in a magnetic field: subtle symmetry and multiparameter bifurcation". J. Elasticity 24, pp. 211-227.

[8] Hughes, P.C., 1986. Spacecraft Attitude Dynamics. Dover Publications, New York.

[9] Jackson, J.D., 1975. Classical Electrodynamics, 2nd edition. John Wiley \& Sons, New York.

[10] Krupa, M., Kuhn, A., Poth, W., Schagerl, M., Steindl, A., Steiner, W., Troger, H. and Wiedermann, G., 2000. "Tethered satellite systems: A new concept of space flight". Eur. J. Mech. A Solids 19, pp. 145-164.

[11] Cosmo, M.L. and Lorenzini, E.C., 1997. Tethers in Space Handbook, 3rd edition. Smithsonian Astrophisical Observatory (NASA Marshall Space Flight Center, Huntsville, Alabama).

[12] Maiya, B.G. and Ramasarma, T., 2001. "DNA, a molecular wire or not - The debate continues". Current Science 80(12), pp. 1523-1530.

[13] Tyc, G., Han, R.P.S., Vigneron, F.R., Jablonski, A.M., Modi, V.J. and Misra, A.K., 1993. "Dynamics and stability of a spinning tethered spacecraft with flexible appendages". Advances in the Astronautical Sciences 85(1), pp. 877-896.

[14] Vigneron, F.R., Jablonski, A.M., Chandrashaker, R. and Tyc, G., 1997. "Damped gyroscopic modes of spinning tethered space vehicles with flexible booms". Journal of Spacecraft and Rockets 34(5), pp. 662-669.

[15] Ahedo, E. and Sanmartín, J.R., 2002. "Analysis of bare-tether systems for deorbiting lowearth-orbit satellites". Journal of Spacecraft and Rockets 39(2), pp. 198-205.

[16] Scheibel, T., Parthasarathy, R., Sawicki, G., Lin, X.M., Jaeger, H. and Lindquist, S.L., 2003. "Conducting nanowires built by controlled self-assembly of amyloid fibers and selective metal 
deposition". Proc. Nat. Acad. Sci. USA 100, pp. 4527-4532.

[17] Sinden, D. and van der Heijden, G.H.M., 2008. "Integrability of a conducting elastic rod in a magnetic field". J. Phys. A: Math. Theor. 41, 045207 (16pp).

[18] Valverde, J., Escalona, J.L., Mayo, J. and Domínguez, J., 2003. "Dynamic analysis of a light structure in outer space: Short Electrodynamic Tether". Multibody System Dynamics 10(1), pp. $125-146$.

[19] Valverde, J., Escalona, J.L., Domínguez, J. and Champneys, A.R., 2006. "Stability and bifurcation analysis of a spinning space tether". Journal of Nonlinear Science 16(5), pp. 507-542.

[20] Valverde, J., Escalona, J.L., Freire, E. and Domínguez, J., 2005. "Stability and bifurcation analysis of a geometrically nonlinear orthotropic Jeffcott model with internal damping". Nonlinear Dynamics 42(2), pp. 137-163.

[21] Wolfe, P., 1983. "Equilibrium states of an elastic conductor in a magnetic field: A paradigm of bifurcation theory". Trans. Am. Math. Soc. 278, pp. 377-387.

[22] Wolfe, P., 1985. "Rotating states of an elastic conductor", in: Physical Mathematics and Nonlinear Partial Differential Equations, J. Lightbourne and S. Rankin (eds), Dekker, New York.

[23] Wolfe, P., 1988. "Bifurcation theory of an elastic conducting rod in a magnetic field". Quart. J. Mech. Appl. Math. 41(2), pp. 265-279.

[24] Woodson, H.H. and Melcher, J.R., 1968. Electromechanical Dynamics, Part II: Fields, Forces, and Motion. John Wiley \& Sons, New York.

[25] Ziegler, S.W. and Cartmell, M.P., 2001. "Using motorized tethers for payload orbital transfer". Journal of Spacecraft and Rockets 38, pp. 904-913. 


\section{Appendix A: Matrices for the linearisation}

The matrices $\boldsymbol{B}_{i}$ appearing in equation (71) are given by

$$
\begin{aligned}
& \boldsymbol{B}_{1}=\left(\begin{array}{ccc}
0 & -\kappa_{3}^{0} & \kappa_{2}^{0} \\
\kappa_{3}^{0} & 0 & -\kappa_{1}^{0} \\
-\kappa_{2}^{0} & \kappa_{1}^{0} & 0
\end{array}\right) \\
& \boldsymbol{B}_{2}=\omega^{2}\left(\begin{array}{lll}
d_{11}^{0} & d_{12}^{0} & 0 \\
d_{21}^{0} & d_{22}^{0} & 0 \\
d_{31}^{0} & d_{32}^{0} & 0
\end{array}\right) \\
& \boldsymbol{B}_{3}=\left(\begin{array}{ccc}
0 & F_{3}^{0} & -F_{2}^{0} \\
-F_{3}^{0} & 0 & F_{1}^{0} \\
F_{2}^{0} & -F_{1}^{0} & 0
\end{array}\right) \\
& \boldsymbol{B}_{4}=\left(\begin{array}{ccc}
F_{2}^{0} \kappa_{2}^{0}+F_{3}^{0} \kappa_{3}^{0}-B\left(d_{22}^{0} d_{11}^{0}-d_{21}^{0} d_{12}^{0}\right) & \left(F_{3}^{0}\right)^{\prime}-F_{1}^{0} \kappa_{2}^{0} & -\left(F_{2}^{0}\right)^{\prime}-F_{1}^{0} \kappa_{3}^{0} \\
-\left(F_{3}^{0}\right)^{\prime}-F_{2}^{0} \kappa_{1}^{0} & F_{3} \kappa_{3}^{0}+F_{1}^{0} \kappa_{1}^{0}-B\left(d_{22}^{0} d_{11}^{0}-d_{21}^{0} d_{12}^{0}\right) & \left(F_{1}^{0}\right)^{\prime}-F_{2}^{0} \kappa_{3}^{0} \\
\left(F_{2}^{0}\right)^{\prime}-F_{3} \kappa_{1}^{0}-B\left(d_{22}^{0} d_{31}^{0}-d_{21}^{0} d_{32}^{0}\right) & -\left(F_{1}^{0}\right)^{\prime}-F_{3}^{0} \kappa_{2}^{0}+B\left(d_{12}^{0} d_{31}^{0}-d_{11}^{0} d_{32}^{0}\right) & F_{1}^{0} \kappa_{1}^{0}+F_{2}^{0} \kappa_{2}^{0}
\end{array}\right), \\
& \boldsymbol{B}_{5}=\left(\begin{array}{lll}
d_{11}^{0} & d_{12}^{0} & d_{13}^{0} \\
d_{21}^{0} & d_{22}^{0} & d_{23}^{0} \\
d_{31}^{0} & d_{32}^{0} & d_{33}^{0}
\end{array}\right), \\
& \boldsymbol{B}_{6}=2 \omega\left(\begin{array}{lll}
d_{12}^{0} & -d_{11}^{0} & 0 \\
d_{22}^{0} & -d_{21}^{0} & 0 \\
d_{32}^{0} & -d_{31}^{0} & 0
\end{array}\right) \text {. }
\end{aligned}
$$

Matrices $\boldsymbol{C}_{i}$ appearing in equation (72) are given by

$$
\begin{gathered}
\boldsymbol{C}_{1}=\boldsymbol{B}_{1}, \\
\boldsymbol{C}_{2}=\left(\begin{array}{ccc}
0 & M_{3}^{0} & -M_{2}^{0} \\
-M_{3}^{0} & 0 & M_{1}^{0} \\
M_{2}^{0} & -M_{1}^{0} & 0
\end{array}\right),
\end{gathered}
$$




$$
\boldsymbol{C}_{3}=\left(\begin{array}{ccc}
C_{3}^{11} & C_{3}^{12} & C_{3}^{13} \\
C_{3}^{21} & C_{3}^{22} & C_{3}^{23} \\
C_{3}^{31} & C_{3}^{32} & C_{3}^{33}
\end{array}\right)
$$

where

$$
\begin{aligned}
& C_{3}^{11}=M_{3}^{0} \kappa_{3}^{0}+M_{2}^{0} \kappa_{2}^{0}-P\left(\boldsymbol{\omega} \cdot \boldsymbol{d}_{3}^{0}\right)\left(\boldsymbol{d}_{2}^{0} \times \boldsymbol{\omega} \cdot \boldsymbol{d}_{1}^{0}\right)-P\left(\boldsymbol{\omega} \cdot \boldsymbol{d}_{2}^{0}\right)\left(\boldsymbol{d}_{3}^{0} \times \boldsymbol{\omega} \cdot \boldsymbol{d}_{1}^{0}\right), \\
& C_{3}^{12}=\left(M_{3}^{0}\right)^{\prime}-M_{1}^{0} \kappa_{2}^{0}-P R\left(\boldsymbol{\omega} \cdot \boldsymbol{d}_{1}^{0}\right)\left(\boldsymbol{d}_{1}^{0} \times \boldsymbol{\omega} \cdot \boldsymbol{d}_{3}^{0}\right), \\
& C_{3}^{13}=-\left(M_{2}^{0}\right)^{\prime}-M_{1}^{0} \kappa_{3}^{0}-F_{1}^{0}+P R\left(\boldsymbol{\omega} \cdot \boldsymbol{d}_{1}^{0}\right)\left(\boldsymbol{d}_{1}^{0} \times \boldsymbol{\omega} \cdot \boldsymbol{d}_{2}^{0}\right)+P\left(\boldsymbol{\omega} \cdot \boldsymbol{d}_{1}^{0}\right)\left(\boldsymbol{d}_{2}^{0} \times \boldsymbol{\omega} \cdot \boldsymbol{d}_{1}^{0}\right), \\
& C_{3}^{21}=-\left(M_{3}^{0}\right)^{\prime}-M_{2}^{0} \kappa_{1}^{0}+P\left(\boldsymbol{\omega} \cdot \boldsymbol{d}_{2}^{0}\right)\left(\boldsymbol{d}_{2}^{0} \times \boldsymbol{\omega} \cdot \boldsymbol{d}_{3}^{0}\right), \\
& C_{3}^{22}=M_{3}^{0} \kappa_{3}^{0}+M_{1}^{0} \kappa_{1}^{0}+P R\left(\boldsymbol{\omega} \cdot \boldsymbol{d}_{3}^{0}\right)\left(\boldsymbol{d}_{1}^{0} \times \boldsymbol{\omega} \cdot \boldsymbol{d}_{2}^{0}\right)+P R\left(\boldsymbol{\omega} \cdot \boldsymbol{d}_{1}^{0}\right)\left(\boldsymbol{d}_{3}^{0} \times \boldsymbol{\omega} \cdot \boldsymbol{d}_{2}^{0}\right), \\
& C_{3}^{23}=\left(M_{1}^{0}\right)^{\prime}-M_{2}^{0} \kappa_{3}^{0}-F_{2}^{0}-P R\left(\boldsymbol{\omega} \cdot \boldsymbol{d}_{2}^{0}\right)\left(\boldsymbol{d}_{1}^{0} \times \boldsymbol{\omega} \cdot \boldsymbol{d}_{2}^{0}\right)-P\left(\boldsymbol{\omega} \cdot \boldsymbol{d}_{2}^{0}\right)\left(\boldsymbol{d}_{2}^{0} \times \boldsymbol{\omega} \cdot \boldsymbol{d}_{1}^{0}\right), \\
& C_{3}^{31}=\left(M_{2}^{0}\right)^{\prime}-M_{3}^{0} \kappa_{1}^{0}+F_{1}^{0}-P\left(\boldsymbol{\omega} \cdot \boldsymbol{d}_{3}^{0}\right)\left(\boldsymbol{d}_{2}^{0} \times \boldsymbol{\omega} \cdot \boldsymbol{d}_{3}^{0}\right), \\
& C_{3}^{32}=-\left(M_{1}^{0}\right)^{\prime}-M_{3}^{0} \kappa_{2}^{0}+F_{2}^{0}+P R\left(\boldsymbol{\omega} \cdot \boldsymbol{d}_{3}^{0}\right)\left(\boldsymbol{d}_{1}^{0} \times \boldsymbol{\omega} \cdot \boldsymbol{d}_{3}^{0}\right), \\
& C_{3}^{33}=M_{2}^{0} \kappa_{2}^{0}+M_{1}^{0} \kappa_{1}^{0}+P(1-R)\left(\boldsymbol{\omega} \cdot \boldsymbol{d}_{2}^{0}\right)\left(\boldsymbol{d}_{1}^{0} \times \boldsymbol{\omega} \cdot \boldsymbol{d}_{3}^{0}\right)+P(1-R)\left(\boldsymbol{\omega} \cdot \boldsymbol{d}_{1}^{0}\right)\left(\boldsymbol{d}_{2}^{0} \times \boldsymbol{\omega} \cdot \boldsymbol{d}_{3}^{0}\right),
\end{aligned}
$$

$$
\begin{gathered}
\boldsymbol{C}_{4}=\left(\begin{array}{ccc}
0 & -1 & 0 \\
1 & 0 & 0 \\
0 & 0 & 0
\end{array}\right), \\
\boldsymbol{C}_{5}=P\left(\begin{array}{lll}
1 & 0 & 0 \\
0 & R & 0 \\
0 & 0 & (1+R)
\end{array}\right),
\end{gathered}
$$

$\boldsymbol{C}_{6}=2 P\left(\begin{array}{ccc}\left(\boldsymbol{d}_{2}^{0} \times \omega \times \boldsymbol{d}_{3}^{0}\right) \cdot \boldsymbol{d}_{1}^{0} & 0 & -\left(\boldsymbol{d}_{2}^{0} \times \omega \times \boldsymbol{d}_{1}^{0}\right) \cdot \boldsymbol{d}_{1}^{0} \\ 0 & -R\left(\boldsymbol{d}_{1}^{0} \times \omega \times \boldsymbol{d}_{3}^{0}\right) \cdot \boldsymbol{d}_{2}^{0} & R\left(\boldsymbol{d}_{1}^{0} \times \omega \times \boldsymbol{d}_{2}^{0}\right) \cdot \boldsymbol{d}_{2}^{0} \\ \left(\boldsymbol{d}_{2}^{0} \times \omega \times \boldsymbol{d}_{3}^{0}\right) \cdot \boldsymbol{d}_{3}^{0}-R\left(\boldsymbol{d}_{1}^{0} \times \omega \times \boldsymbol{d}_{3}^{0}\right) \cdot \boldsymbol{d}_{3}^{0} & R\left(\boldsymbol{d}_{1}^{0} \times \omega \times \boldsymbol{d}_{2}^{0}\right) \cdot \boldsymbol{d}_{3}^{0}-\left(\boldsymbol{d}_{2}^{0} \times \omega \times \boldsymbol{d}_{1}^{0}\right) \cdot \boldsymbol{d}_{3}^{0}\end{array}\right)$

Matrices $\boldsymbol{D}_{i}$ appearing in equation (174) are given by 


$$
\begin{aligned}
& \boldsymbol{D}_{1}=\frac{1}{f}\left(\begin{array}{ccc}
-1 & 0 & 0 \\
0 & -R & 0 \\
0 & 0 & -\frac{\Gamma(1+R)}{2}
\end{array}\right) \\
& \boldsymbol{D}_{2}=\left(\begin{array}{ccc}
0 & \frac{1}{f} \kappa_{3}^{0} & -M_{2}^{0}-\frac{1}{f}(1-R) \kappa_{2}^{0} \\
-\frac{1}{f} \kappa_{3}^{0} & 0 & M_{1}^{0}-\frac{1}{f}(1-R) \kappa_{1}^{0} \\
M_{2}^{0}-\frac{1}{f} \kappa_{2}^{0}\left(R-\frac{\Gamma(1+R)}{2}\right) & -M_{1}^{0}+\frac{1}{f} \kappa_{1}^{0}\left(1-\frac{\Gamma(1+R)}{2}\right) & 0
\end{array}\right), \\
& \boldsymbol{D}_{3}=\frac{\gamma}{f}\left(\begin{array}{ccc}
0 & -\kappa_{3}^{0} & \kappa_{2}^{0} \\
R \kappa_{3}^{0} & 0 & -R \kappa_{1}^{0} \\
-\frac{\Gamma(1+R)}{2} \kappa_{2}^{0} & \frac{\Gamma(1+R)}{2} \kappa_{1}^{0} & 0
\end{array}\right) \\
& \boldsymbol{D}_{4}=-\gamma \boldsymbol{D}_{1} \text {. }
\end{aligned}
$$

All the $\kappa_{i}^{0}$ in the above can be expressed in terms of the moments $M_{i}^{0}$ by means of the constitutive relations (57). 